\title{
$G$-surgery on 3-dimensional Manifolds for Homology Equivalences
}

\author{
By
}

\author{
Masaharu MoRimoto*
}

\begin{abstract}
For a finite group $G$ and a $G$-map $f: X \rightarrow Y$ of degree one, where $X$ and $Y$ are compact, connected, oriented, 3-dimensional, smooth $G$-manifolds, we give an obstruction element $\sigma(f)$ in a $K$-theoretic group called the Bak group, with the property: $\sigma(f)=0$ guarantees that one can perform $G$-surgery on $X$ so as to convert $f$ to a homology equivalence $f^{\prime}: X^{\prime} \rightarrow Y$. Using this obstruction theory, we determine the $G$-homeomorphism type of the singular set of a smooth action of $A_{5}$ on a 3 -dimensional homology sphere having exactly one fixed point, where $A_{5}$ is the alternating group on five letters.
\end{abstract}

\section{$\S 1 . \quad$ Introduction}

This paper is a continuation of [7]. For a finite group $G$, we discuss $G$ equivariant surgery on compact connected oriented 3-dimensional manifolds, and construct an algebraic obstruction to converting a framed $G$-map of degree one to a homology equivalence by a finite sequence of $G$-surgeries of free orbit type. The purpose of the current paper is to improve [2, Theorem 1] to Theorem 1.1 below. Moreover, we give a detailed proof of this theorem in the present paper, while [2] omits the details of the proof. In [2], as well as in [7, p.78], we exhibited the importance of 3-dimensional $G$-surgery theory from the

Communicated by Y. Miyaoka, June 6, 2000. Revised September 11, 2000.

2000 Mathematics Subject Classification(s): 57R91, 57R67, 57R19.

Key words and phrases: 3-dimensional manifolds, equivariant surgery, Bak groups, 3dimensional homology spheres.

Partiall supported by Grant-in-Aid for Scientific Research.

* Department of Environmental and Mathematical Sciences, Faculty of Environmental Science and Technology, Okayama University, Tsushimanaka, Okayama, 700-8530 Japan.

e-mail: morimoto@ems.okayama-u.ac.jp 
viewpoint of smooth actions of $G$ on spheres. In particular, the theory is a key tool constructing smooth actions of $A_{5}$ on spheres of dimensions 7 and 8 with exactly one fixed point, and we also apply it in Section 5 to determine the $G$-homeomorphism type of the singular set of a smooth action of $A_{5}$ on a 3-dimensional homology sphere with exactly one fixed point.

Let $G$ be a finite group, $e \in G$ the identity element, and set

$$
G(2)=\left\{g \in G \mid g^{2}=e, g \neq e\right\} .
$$

All manifolds are understood to be paracompact smooth manifolds, and $G$ actions to be smooth, unless otherwise stated.

For a compact $G$-manifold $X$, we define the singular set $X_{s}$ (or more precisely $\left.X_{s(G)}\right)$ by

$$
X_{s}=\bigcup_{g \in G \backslash\{e\}} X^{g},
$$

where $X^{g}$ is the fixed point set of $g$ in $X$. In the case $\operatorname{dim} X=3$, we define

$$
G(X)=\left\{g \in G(2) \mid \operatorname{dim} X^{g}=1\right\}
$$

Here $\operatorname{dim} X^{g}$ is the maximal dimension of connected components of $X^{g}$. We denote by $\mathcal{M}^{3}(G)$ the family of all compact connected oriented 3-dimensional $G$-manifolds $X$ (possibly with boundary $\partial X$ ) satisfying

$$
\operatorname{dim} X_{s(G)} \leq 1
$$

In [2], we assumed the additional condition

$$
G(X)=G(2)
$$

but this is not necessary in the current paper.

Let $Y \in \mathcal{M}^{3}(G)$. Then the orientation homomorphism $w=w_{Y}: G \rightarrow$ $\{ \pm 1\}$ is defined by $w(g)=1$ if $g \in G$ preserves the orientation of $Y$, and -1 otherwise. For a commutative $\operatorname{ring} R$ with identity, the group $\operatorname{ring} R[G]$ of $G$ over $R$ is defined to be the set of all formal sums $\sum_{g \in G} r_{g} g$, with $r_{g} \in R$. The group ring $R[G]$ has the involution - associated with $w_{Y}$ which is defined by

$$
\left(\sum_{g \in G} r_{g} g\right)^{-}=\sum_{g \in G} r_{g} w_{Y}(g) g^{-1} .
$$

We denote by $\tilde{Y}$ the universal covering space of $Y$. A 1-connected $G$-map $f: X \rightarrow Y$ (hence $\pi_{1}(f): \pi_{1}(X) \rightarrow \pi_{1}(Y)$ is surjective) induces the covering 
space $\widetilde{X}=f^{*} \tilde{Y}$ of $X$ from $\tilde{Y}$ and the map $\tilde{f}: \widetilde{X} \rightarrow \tilde{Y}$ covering $f$, giving the pullback diagram

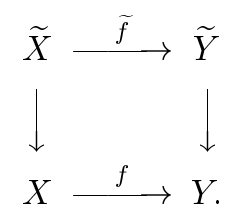

The group $\widetilde{G}=\pi_{1}\left(E G \times_{G} Y\right)$ is an extension of $G$ by $\pi_{1}(Y)$ :

$$
\{e\} \rightarrow \pi_{1}(Y) \rightarrow \widetilde{G} \rightarrow G \rightarrow G / G \quad \text { (exact) }
$$

and acts on $\widetilde{Y}$ and $\widetilde{X}$ (cf. [8]). With respect to the $\widetilde{G}$-actions, $\widetilde{f}$ is a $\widetilde{G}$-map.

We say that a finite group $H$ is p-hyperelementary (for $p$ a prime) if $H$ is an extension of a $p$-group by a cyclic group: $H=C \rtimes P$, where $C$ is cyclic and $P$ is of order $p^{n}$ for some nonnegative integer $n$. A hyperelementary group means a $p$-hyperelementary group for some prime $p$.

Theorem 1.1. Let $R$ be a ring such that $\mathbb{Z} \subset R \subset \mathbb{Q}$. Suppose that $X$ and $Y$ in $\mathcal{M}^{3}(G)$ satisfy

$$
G(X)=G(Y)
$$

and $f:(X, \partial X) \rightarrow(Y, \partial Y)$ is a 1-connected $G$-map of degree one such that

(1.1.1) $\partial f=\left.f\right|_{\partial X}: \partial X \rightarrow \partial Y$ is a homotopy equivalence, and

(1.1.2) $f_{s(H)}: X_{s(H)} \rightarrow Y_{s(H)}$ is an R-homology equivalence for each hyperelementary subgroup $H$ of $G$.

Moreover, let $b: T(X) \oplus f^{*} \eta_{-} \rightarrow \eta_{+}$be an orientation preserving map of $G$ vector bundles covering $f$, where $\eta_{+}$and $\eta_{-}$are oriented real $G$-vector bundles over $Y$ such that $\eta_{+} \supset \varepsilon_{Y}\left(\mathbb{R}^{4}\right)$. Then there exists an obstruction element $\sigma(f, b)$ in the Bak group $W_{3}\left(R[G], \Gamma G(Y) ; w_{Y}\right)$ (cf. [5], [2]), where $\Gamma G(Y)$ is the smallest form parameter on $R[G]$ containing $G(Y)$, with the property that if $\sigma(f, b)=0$ then one can perform $G$-surgery on $X \backslash\left(\partial X \cup X_{s(G)}\right)$ to alter $f: X \rightarrow Y$ a G-map $f^{\prime}: X^{\prime} \rightarrow Y$ which is an R-homology equivalence, and $b: T(X) \oplus f^{*} \eta_{-} \rightarrow \eta_{+}$to a map $b^{\prime}: T\left(X^{\prime}\right) \oplus f^{\prime *} \eta_{-} \rightarrow \eta_{+}$of G-vector bundles covering $f^{\prime}$.

Remark 1.2. If the reduced projective class group $\widetilde{K}_{0}(\mathbb{Z}[G])$ is trivial, then (1.1.2) can be replaced by the condition that

(1.2.1) $f^{P}: X^{P} \rightarrow Y^{P}$ is $\bmod p$ homology equivalence for each prime $p$ dividing $|G|$ and every nontrivial $p$-subgroup $P$ of $G$. 
Theorem 1.1 improves [2, Theorem 1] in two respects. One is that the condition $(\mathcal{M} 2)$ is removed, and the other is that $Y$ is not restricted to be simply connected.

In the case without bundle data, we have

Theorem 1.3. Let $R$ be a ring such that $\mathbb{Z} \subset R \subset \mathbb{Q}, X$ and $Y$ in $\mathcal{M}^{3}(G)$, and $f:(X, \partial X) \rightarrow(Y, \partial Y)$ a 1-connected $G$-map of degree one satisfying the conditions (1.1.1), (1.1.2) and (M2) above. Then, there is an obstruction element $\sigma(f)$ in the Bak group $W_{3}(R[G]$, max; triv), where max is the maximal form parameter on $R[G]$ and triv is the trivial homomorphism $G \rightarrow\{1\}$, with the property that if $\sigma(f)=0$ then one can perform $G$-surgery on $X \backslash\left(\partial X \cup X_{s(G)}\right)$ to a $G$-map $f^{\prime}: X^{\prime} \rightarrow Y$ which is an $R$-homology equivalence.

For applications of Theorems 1.1 and 1.3, the results of A. Bak, e.g., [2, Theorems 3-5 and Corollary 6], are quite useful, since they guarantee that the $G$-surgery obstruction vanishes.

The organization of the rest of this paper is as follows. Section 2 treats algebraic preliminaries, including the definition of quadratic forms and $G$-surgery obstruction groups. The equality in Proposition 2.9 is a key to surgery theory on odd dimensional manifolds. In Section 3, we argue how we assign the $G$-surgery obstruction $\sigma(f)$ to a $G$-map $f: X \rightarrow Y$ of degree one satisfying certain conditions. We prove Theorem 1.1 in Section 4 . Namely we prove that $\sigma(f)=0$ guarantees the existence of a finite sequence of $G$-surgeries converting $f$ to a $G$-map $f^{\prime}: X^{\prime} \rightarrow Y$ which is an $R$-homology equivalence. In Section 5 , we give an application of our $G$-surgery theory concerned with the singular sets of smooth actions of $A_{5}$ on 3-dimensional homology spheres.

\section{$\S 2 . \quad$ Quadratic Modules and the Bak Groups}

In the current section we recall the definition of form parameters, quadratic modules and the Bak groups. If the reader is familiar with [2] or [5] then he can skip the section.

Let $A$ be a ring with identity. We always suppose that a finitely generated free $A$-module has a well-defined rank over $A$, i.e., if $A^{m} \cong A^{n}$ then $m=n$. Let - be an involution on $A$ such that

(i) $\overline{\bar{a}}=a \quad(a \in A)$,

(ii) $\overline{a+b}=\bar{a}+\bar{b} \quad(a, b \in A)$, 
(iii) $\overline{a b}=\bar{b} \bar{a} \quad(a, b \in A)$ and

(iv) $\overline{1}=1$.

Let $s \in \operatorname{Center}(A)$ such that $s \bar{s}=1$. This element $s$ is called a symmetry of $A$. Then a form parameter $\Gamma$ on $A$ is defined to be an additive subgroup of $A$ such that

(Г1) $\{a-s \bar{a} \mid a \in A\} \subset \Gamma \subset\{a \in A \mid a=-s \bar{a}\}$ and

(Г2) $a \Gamma \bar{a} \subset \Gamma$ for all $a \in A$.

The maximal and minimal choices are denoted by max and min respectively, i.e.,

$$
\max =\{a \in A \mid a=-s \bar{a}\} \quad \text { and } \quad \min =\{a-s \bar{a} \mid a \in A\} .
$$

In the following, quadratic forms and modules are defined depending on the datum

$$
\boldsymbol{A}=(A,-, s, \Gamma)
$$

called a form ring. Let $M$ be a left $A$-module. A sesquilinear form on $M$ is a biadditive map

$$
B: M \times M \rightarrow A
$$

such that

$$
B(a x, b y)=b B(x, y) \bar{a} \quad(a, b \in A, x, y \in M) .
$$

A sesquilinear form $B$ is called $s$-Hermitian if

$$
B(x, y)=s \overline{B(y, x)} \quad(x, y \in M) .
$$

Definition 2.1. A quadratic $\boldsymbol{A}$-module is defined to be a triple $(M, B, q)$ consisting of a finitely generated projective $A$-module $M$, an $s$-Hermitian form $B: M \times M \rightarrow A$ and a map $q: M \rightarrow A / \Gamma$ which satisfy the following conditions $(2.1 .1)-(2.1 .3):$

(2.1.1) $q(a x)=a q(x) \bar{a}(a \in A, x \in M)$,

(2.1.2) $q(x+y)-q(x)-q(y) \equiv B(x, y) \quad \bmod \Gamma(x, y \in M)$ and

(2.1.3) $\widetilde{q}(x)+s \overline{\widetilde{q}(x)}=B(x, x)(x \in M)$ for any lifting $\widetilde{q}(x) \in A$ of $q(x) \in A / \Gamma$.

The map $q: M \rightarrow A / \Gamma$ above is called a $\Gamma$-quadratic form. 
A morphism $(M, B, q) \rightarrow\left(M^{\prime}, B^{\prime}, q^{\prime}\right)$ of quadratic $\boldsymbol{A}$-modules is an $A$-linear map $M \rightarrow M^{\prime}$ which preserves both Hermitian and quadratic forms. We say that a quadratic $\boldsymbol{A}$-module $(M, B, q)$ is nonsingular if the Hermitian form $B$ is nonsingular, i.e., the map

$$
M \rightarrow \operatorname{Hom}_{A}(M, A) ; x \mapsto B(x, \quad)
$$

is bijective.

Definition 2.2. $\quad$ Define the standard hyperbolic $\boldsymbol{A}$-module $\mathbf{H}_{m}$ of rank $2 m$ to be the quadratic $\boldsymbol{A}$-module $\left(A^{2 m}, B, q\right)$ such that

$$
\begin{aligned}
& A^{2 m} \text { is a free } A \text {-module with basis }\left\{e_{1}, \ldots, e_{m}, f_{1}, \ldots, f_{m}\right\}, \\
& B\left(\sum_{i}\left(a_{i} e_{i}+b_{i} f_{i}\right), \sum_{i}\left(a_{i}^{\prime} e_{i}+b_{i}^{\prime} f_{i}\right)\right)=\sum_{i}\left(b_{i}^{\prime} \overline{a_{i}}+s a_{i}^{\prime} \overline{b_{i}}\right) \text { and } \\
& q\left(\sum_{i} a_{i} e_{i}+b_{i} f_{i}\right)=\left[\sum_{i} b_{i} \overline{a_{i}}\right] \text { in } A / \Gamma,
\end{aligned}
$$

where $a_{i}, b_{i}, a_{i}^{\prime}, b_{i}^{\prime} \in A$.

Let $M$ and $M^{\prime}$ be free $A$-modules with ordered bases $\left\{x_{1}, \ldots, x_{n}\right\}$ and $\left\{y_{1}, \ldots, y_{\ell}\right\}$. For an $A$-homomorphism $f: M \rightarrow M^{\prime}$, we obtain a matrix $\operatorname{Mat}(f)=\left(m_{i j}\right)$ (or more precisely $\left.\operatorname{Mat}\left(f ;\left\{x_{i}\right\},\left\{y_{j}\right\}\right)\right)$ by

$$
f\left(x_{i}\right)=\sum_{j=1}^{\ell} m_{i j} y_{j}
$$

for each $i=1, \ldots, n$. We use the notation

$$
f\left(\begin{array}{c}
x_{1} \\
\vdots \\
x_{n}
\end{array}\right) \text { for } \quad\left(\begin{array}{c}
f\left(x_{1}\right) \\
\vdots \\
f\left(x_{n}\right)
\end{array}\right)
$$

Then, we can express the relation between $f$ and Mat $(f)$ by

$$
f\left(\begin{array}{c}
x_{1} \\
\vdots \\
x_{n}
\end{array}\right)=\operatorname{Mat}(f)\left(\begin{array}{c}
y_{1} \\
\vdots \\
y_{\ell}
\end{array}\right) .
$$

Proposition 2.3 (cf. [3, p.37, Beware]). Let $M=\left\langle x_{1}, \ldots, x_{n}\right\rangle_{A}$ be as above and $f, g \in \operatorname{End}_{A}(M)$. Then $\operatorname{Mat}(f g)$ is equal to $\operatorname{Mat}(g) \operatorname{Mat}(f)$. 
Let $A^{2 m}=\left\langle e_{1}, \ldots, e_{m}, f_{1}, \ldots, f_{m}\right\rangle_{A}$ as before and $f \in \operatorname{End}_{A}\left(A^{2 m}\right)$. Then the matrix Mat $(f)$ associated with $f$ is expressed in the form

$$
\operatorname{Mat}(f)=\left(\begin{array}{ll}
M_{11} & M_{12} \\
M_{21} & M_{22}
\end{array}\right),
$$

using $m \times m$-matrices $M_{11}=\left(a_{i j}\right), M_{12}=\left(b_{i j}\right), M_{21}=\left(c_{i j}\right), M_{22}=\left(d_{i j}\right)$.

Proposition 2.4. An element $f \in \operatorname{End}_{A}\left(A^{2 m}\right)$ is an automorphism of $\mathbf{H}_{m}$ if and only if the following (2.4.1)-(2.4.3) are satisfied:

$$
\begin{aligned}
& \text { (2.4.1) }\left(\begin{array}{ll}
M_{11} & M_{12} \\
M_{21} & M_{22}
\end{array}\right) \in \mathrm{GL}_{2 m}(A) . \\
& (2.4 .2)\left(\begin{array}{ll}
M_{11} & M_{12} \\
M_{21} & M_{22}
\end{array}\right)^{-1}=\left(\begin{array}{cc}
{ }^{t} \overline{M_{22}} & s^{t} \overline{M_{12}} \\
\bar{s}^{t} \overline{M_{21}} & t^{t} \overline{M_{11}}
\end{array}\right) .
\end{aligned}
$$

(2.4.3) The diagonal coefficients of $M_{12}\left({ }^{t} \overline{M_{11}}\right)$ and $M_{22}\left({ }^{t} \overline{M_{21}}\right)$ lie in $\Gamma$.

Proof. We prove the only if part. Let $f$ be an automorphism of $\mathbf{H}_{m}$. Then (2.4.1) clearly holds. Observe the relations:

$$
\begin{aligned}
& B\left(f\left(e_{i}\right), f\left(e_{j}\right)\right)=\sum_{k}\left(b_{j k} \overline{a_{i k}}+s a_{j k} \overline{b_{i k}}\right)=0, \\
& B\left(f\left(f_{i}\right), f\left(f_{j}\right)\right)=\sum_{k}\left(d_{j k} \overline{c_{i k}}+s c_{j k} \overline{d_{i k}}\right)=0, \\
& B\left(f\left(e_{i}\right), f\left(f_{j}\right)\right)=\sum_{k}\left(d_{j k} \overline{a_{i k}}+s c_{j k} \overline{b_{i k}}\right)=\delta_{i j} \text { and } \\
& B\left(f\left(f_{i}\right), f\left(e_{j}\right)\right)=\sum_{k}\left(b_{j k} \overline{c_{i k}}+s a_{j k} \overline{d_{i k}}\right)=s \delta_{i j} .
\end{aligned}
$$

It follows that

$$
\begin{aligned}
\left(\begin{array}{ll}
M_{11} & M_{12} \\
M_{21} & M_{22}
\end{array}\right)\left(\begin{array}{cl}
{ }^{t} \overline{M_{22}} & s^{t} \overline{M_{12}} \\
\bar{s}^{t} \overline{M_{21}} & { }^{t} \overline{M_{11}}
\end{array}\right) \\
=\left(\begin{array}{ll}
\left(a_{i j}\right) & \left(b_{i j}\right) \\
\left(c_{i j}\right) & \left(d_{i j}\right)
\end{array}\right)\left(\begin{array}{cc}
\left(\overline{d_{j i}}\right) & s\left(\overline{b_{j i}}\right) \\
\bar{s}\left(\overline{c_{j i}}\right) & \left(\overline{a_{j i}}\right)
\end{array}\right) \\
=\left(\begin{array}{ll}
\sum_{k}\left(a_{i k} \overline{d_{j k}}+\bar{s} b_{i k} \overline{c_{j k}}\right) & \sum_{k}\left(s a_{i k} \overline{b_{j k}}+b_{i k} \overline{a_{j k}}\right) \\
\sum_{k}\left(c_{i k} \overline{d_{j k}}+\bar{s} d_{i k} \overline{c_{j k}}\right) & \sum_{k}\left(s c_{i k} \overline{b_{j k}}+d_{i k} \overline{a_{j k}}\right)
\end{array}\right) \\
=\left(\begin{array}{ll}
I_{m} & 0_{m} \\
0_{m} & I_{m}
\end{array}\right) .
\end{aligned}
$$


This proves (2.4.2). The final condition (2.4.3) follows from the equalities

$$
\begin{aligned}
& q\left(f\left(e_{i}\right)\right)=\left[\sum_{k} b_{i k} \overline{a_{i k}}\right]=0 \text { in } A / \Gamma \text { and } \\
& q\left(f\left(f_{i}\right)\right)=\left[\sum_{k} d_{i k} \overline{c_{i k}}\right]=0 \text { in } A / \Gamma .
\end{aligned}
$$

The if part also follows from the equalities above.

Q.E.D.

For a $2 m \times 2 m$-matrix $M=\left(\begin{array}{ll}M_{11} & M_{12} \\ M_{21} & M_{22}\end{array}\right)$ and $2 m^{\prime} \times 2 m^{\prime}$-matrix $M^{\prime}=$ $\left(\begin{array}{cc}M_{11}^{\prime} & M_{12}^{\prime} \\ M_{21}^{\prime} & M_{22}^{\prime}\end{array}\right)$, we define the $2\left(m+m^{\prime}\right) \times 2\left(m+m^{\prime}\right)$-matrix $M \oplus M^{\prime}$ to be

$$
\left(\begin{array}{cccc}
M_{11} & 0 & M_{12} & 0 \\
0 & M_{11}^{\prime} & 0 & M_{12}^{\prime} \\
M_{21} & 0 & M_{22} & 0 \\
0 & M_{21}^{\prime} & 0 & M_{22}^{\prime}
\end{array}\right) .
$$

We define several matrix groups which will be needed later. Firstly, define

$$
\mathrm{SU}_{m}(A, \Gamma)=\left\{\left(\begin{array}{ll}
M_{11} & M_{12} \\
M_{21} & M_{22}
\end{array}\right) \in \mathrm{M}_{2 m, 2 m}(A) \mid(2.4 .1)-(2.4 .3) \text { are satisfied }\right\} \text {. }
$$

This contains the subgroup

$$
\mathrm{TU}_{m}(A, \Gamma)=\left\{\left(\begin{array}{ll}
M_{11} & M_{12} \\
M_{21} & M_{22}
\end{array}\right) \in \mathrm{SU}_{m}(A, \Gamma) \mid M_{12}=0\right\} .
$$

We define the $2 \times 2$-matrix $\sigma$ by

$$
\sigma=\left(\begin{array}{ll}
0 & 1 \\
\bar{s} & 0
\end{array}\right)
$$

Then $\sigma$ lies in $\operatorname{SU}_{1}(A, \Gamma)$. We set $\sigma_{i}=I_{2(i-1)} \oplus \sigma \oplus I_{2(m-i)}$ and

$$
\mathrm{RU}_{m}(A, \Gamma)=\left\langle\mathrm{TU}_{m}(A, \Gamma), \sigma_{1}, \ldots, \sigma_{m}\right\rangle\left(\subset \mathrm{SU}_{m}(A, \Gamma)\right) .
$$

The stabilization homomorphism

$$
j_{m, m+1}: \mathrm{SU}_{m}(A, \Gamma) \rightarrow \mathrm{SU}_{m+1}(A, \Gamma)
$$

is defined by $j_{m, m+1}(M)=M \oplus I_{2}$. 
A matrix having one form among the following $2 m \times 2 m$-matrices $\varepsilon_{* *}(\quad)$ and $\mathbf{H}\left(\varepsilon_{* *}(\quad)\right.$ ) (for some $m$ ) is called a $\Gamma$-quadratic elementary matrix.

$$
\begin{gathered}
\varepsilon_{m+i, j}(a)(i \neq j, a \in A):\left\{\begin{array}{l}
\text { the }(k, k) \text {-entry }=1(k=1, \ldots, 2 m), \\
\text { the }(m+i, j) \text {-entry }=a, \\
(m+j, i) \text {-entry }=-\overline{s a}, \\
\text { all other entries }=0 .
\end{array}\right. \\
\varepsilon_{i, m+j}(a)(i \neq j, a \in A): \quad\left\{\begin{array}{l}
\text { the }(k, k) \text {-entry }=1(k=1, \ldots, 2 m), \\
\text { the }(i, m+j) \text {-entry }=a, \\
(j, m+i) \text {-entry }=-s \bar{a}, \\
\text { all other entries }=0 .
\end{array}\right. \\
\varepsilon_{m+i, i}(a)(a \in \bar{\Gamma}): \quad\left\{\begin{array}{l}
\text { the }(k, k) \text {-entry }=1(k=1, \ldots, 2 m), \\
\text { the }(m+i, i) \text {-entry }=a, \\
\text { all other entries }=0 .
\end{array}\right. \\
\varepsilon_{i, m+i}(a)(a \in \Gamma): \quad\left\{\begin{array}{l}
\text { the }(k, k) \text {-entry }=1(k=1, \ldots, 2 m), \\
\text { the }(i, m+i) \text {-entry }=a, \\
\text { all other entries }=0 .
\end{array}\right. \\
\mathbf{H}\left(\varepsilon_{i, j}(a)\right)(i \neq j, a \in A): \quad\left\{\begin{array}{l}
\text { the }(k, k) \text {-entry }=1(k=1, \ldots, 2 m), \\
\text { the }(i, j) \text {-entry }=a, \\
(m+j, m+i) \text {-entry }=-\bar{a}, \\
\text { all other entries }=0 .
\end{array}\right.
\end{gathered}
$$

Using these $\Gamma$-quadratic elementary matrices, we define

$$
\begin{aligned}
\mathrm{EU}_{m}(A, \Gamma)= & \left\langle\text { all elementary matrices } \in \mathrm{SU}_{m}(A, \Gamma)\right\rangle, \text { and } \\
\mathrm{FU}_{m}(A, \Gamma)= & \left\langle\text { all elementary matrices } \in \mathrm{SU}_{m}(A, \Gamma) \text { of type } \varepsilon_{m+i, j}(a)\right. \\
& \left.(i \neq j, a \in A) \text { or } \varepsilon_{m+i, i}(a)(a \in \Gamma), \text { and } \sigma_{1}, \ldots, \sigma_{m}\right\rangle .
\end{aligned}
$$

For $\mathrm{L}=\mathrm{E}, \mathrm{F}, \mathrm{R}, \mathrm{S}, \mathrm{T}$, we set

$$
\mathrm{LU}(A, \Gamma)=\underset{m}{\lim } \operatorname{LU} m(A, \Gamma) .
$$

By [1, Corollary 3.9], we obtain

Lemma 2.5. It holds that $\mathrm{EU}(A, \Gamma)=[\mathrm{SU}(A, \Gamma), \mathrm{SU}(A, \Gamma)]$ the commutator subgroup of $\mathrm{SU}(A, \Gamma)$.

It is easy to see

$$
\mathrm{RU}(A, \Gamma) \supset \mathrm{EU}(A, \Gamma) .
$$

Thus, the quotient group $\mathrm{SU}(A, \Gamma) / \mathrm{RU}(A, \Gamma)$ is abelian. 
Definition 2.6. The Bak group $W_{1}^{s}(A, \Gamma)$, where $s$ is the symmetry of $A$, is defined to be the quotient group $\operatorname{SU}(A, \Gamma) / \mathrm{RU}(A, \Gamma)$. For a commutative ring $R$ with identity, $W_{3}(R[G], \Gamma ; w)$ stands for $W_{1}^{-1}(R[G], \Gamma)$, where the involution - on $R[G]$ is one induced by the orientation homomorphism $w: G \rightarrow\{ \pm 1\}$.

We obtain the next proposition by straightforward calculation.

Proposition 2.7. If $\tau(\quad)=\varepsilon_{* *}(\quad)$ is a $\Gamma$-quadratic elementary matrix, then

$$
\tau(a) \tau(b)=\tau(a+b)
$$

If $i, j$ and $k$ are distinct, then

$$
\begin{aligned}
{\sigma_{i}}^{-1}{\sigma_{j}}^{-1} \varepsilon_{m+i, j}(a) \sigma_{i} \sigma_{j} & =\varepsilon_{i, m+j}(\bar{s} a) \quad(a \in A), \\
\sigma_{i}{ }^{-1} \varepsilon_{m+i, i}(a) \sigma_{i} & =\varepsilon_{i, m+i}(s a) \quad(a \in \bar{\Gamma}), \\
{\left[\varepsilon_{i, m+j}(1), \varepsilon_{m+i, k}(a)\right] } & =\mathbf{H}\left(\varepsilon_{j k}(-s a)\right) \quad(a \in A), \\
{\sigma_{j}}^{-1} \varepsilon_{m+i, j}(a) \sigma_{j} & =\mathbf{H}\left(\varepsilon_{j i}(-\bar{a})\right) \quad(a \in A),
\end{aligned}
$$

where $[x, y]=x^{-1} y^{-1} x y$.

This proposition clearly implies the following two.

Proposition 2.8. Provided $m \geq 2$, it holds that $\mathrm{FU}_{m}(A, \Gamma) \supset$ $\mathrm{EU}_{m}(A, \Gamma)$.

Proposition 2.9. For each element $x \in \mathrm{SU}(A, \Gamma)$, it holds that

$$
\mathrm{TU}(A, \Gamma) x \operatorname{FU}(A, \Gamma)=x \operatorname{RU}(A, \Gamma)=\operatorname{RU}(A, \Gamma) x
$$

as subsets of $\mathrm{SU}(A, \Gamma)$.

Let $(M, B, q)$ be a quadratic $\boldsymbol{A}$-module. We say that a submodule $N$ of $M$ is totally isotropic if $B(x, y)=0$ for all $x, y \in N$ and if $q(x)=0$ for all $x \in N$.

Proposition 2.10. Let $\left(A^{2 m}, B, q\right)$ be a nonsingular quadratic $\boldsymbol{A}$ module. If a direct summand $N$ of $M=A^{2 m}$ is a free $A$-module with basis $\left\{x_{1}, \ldots, x_{m}\right\}$ and is totally isotropic, then there exists a totally isotropic complementary direct summand $L$ with $A$-basis $\left\{y_{1}, \ldots, y_{m}\right\}$ such that $B\left(x_{i}, y_{j}\right)=\delta_{i j}$ for all $i$ and $j$. 
Proof. Let $L^{\prime}$ be a direct summand complementary to $N$ in $M$, i.e., $N \oplus$ $L^{\prime}=M$. We denote by $p$ the projection from $M$ to $L^{\prime}$, and by $f$ the inclusion of $N$ to $M$. The Hermitian form $B$ induces an $A$-homomorphism

$$
\Psi: M \rightarrow M^{\#}=\operatorname{Hom}_{A}(M, A) ; x \mapsto B(x,) .
$$

Since $B$ is nonsingular, $\Psi$ is an isomorphism. It is easy to see that $f^{\#} \Psi: L^{\prime} \rightarrow$ $N^{\#}$ is an isomorphism, because the rank over $A$ is well-defined. There exist $z_{i} \in M, i=1, \ldots, m$, such that $B\left(z_{i}, x_{j}\right)=s \delta_{i j}$ (equally $B\left(x_{i}, z_{j}\right)=\delta_{i j}$ ). Here we note that $\left\{p\left(z_{1}\right), \ldots, p\left(z_{m}\right)\right\}$ is a basis of $L^{\prime}$. Let us make an inductive assumption that $B\left(z_{i}, z_{j}\right)=0$ and $q\left(z_{i}\right)=0$ for all $i, j \leq k$. Set

$$
z_{k+1}^{\prime}=z_{k+1}-\bar{s}\left(\sum_{i=1}^{k} B\left(z_{i}, z_{k+1}\right) x_{i}+\tilde{q}\left(z_{k+1}\right) x_{k+1}\right),
$$

where $\tilde{q}\left(z_{k+1}\right) \in A$ is a lifting of $q\left(z_{k+1}\right) \in A / \Gamma$. If $i \leq k$, then

$$
B\left(z_{i}, z_{k+1}^{\prime}\right)=B\left(z_{i}, z_{k+1}\right)-\bar{s} B\left(z_{i}, z_{k+1}\right) s=0 .
$$

Furthermore,

$$
\begin{aligned}
q\left(z_{k+1}^{\prime}\right)= & q\left(z_{k+1}\right)+q\left(-\bar{s}\left(\sum_{i=1}^{k} B\left(z_{i}, z_{k+1}\right) x_{i}+\tilde{q}\left(z_{k+1}\right) x_{k+1}\right)\right) \\
& +B\left(z_{k+1},-\bar{s}\left(\sum_{i=1}^{k} B\left(z_{i}, z_{k+1}\right) x_{i}+\tilde{q}\left(z_{k+1}\right) x_{k+1}\right)\right) \\
= & q\left(z_{k+1}\right)-\bar{s} \tilde{q}\left(z_{k+1}\right) B\left(z_{k+1}, x_{k+1}\right) \\
= & q\left(z_{k+1}\right)-\tilde{q}\left(z_{k+1}\right) \\
= & 0 \quad \text { in } A / \Gamma
\end{aligned}
$$

and

$$
B\left(z_{k+1}^{\prime}, z_{k+1}^{\prime}\right)=\tilde{q}\left(z_{k+1}^{\prime}\right)+s \overline{\tilde{q}\left(z_{k+1}^{\prime}\right)}=0
$$

because of the condition $(\Gamma 2)$ and $\tilde{q}\left(z_{k+1}^{\prime}\right) \in \Gamma$. Thus, there exist $y_{i} \in M$, $i=1, \ldots, m$, such that $B\left(x_{i}, y_{j}\right)=\delta_{i j}, B\left(y_{i}, y_{j}\right)=0$ and $q\left(y_{i}\right)=0$. We set $L=\left\langle y_{1}, \ldots, y_{m}\right\rangle_{A}$. Since $\left\{p\left(y_{1}\right), \ldots, p\left(y_{m}\right)\right\}$ is a basis of $L^{\prime}, M$ is the direct sum of $N$ and $L$.

Q.E.D.

We close this section by remarking

$$
\mathrm{RU}_{m}(A, \Gamma)=\left\langle\mathrm{TU}_{m}(A, \Gamma), \sigma_{1}\right\rangle
$$

as subsets of $\mathrm{SU}_{m}(A, \Gamma)$. 


\section{$\S 3 . \quad$ Definition of $G$-surgery Obstructions}

Let $X$ and $Y$ be elements in $\mathcal{M}^{3}(G), f:(X, \partial X) \rightarrow(Y, \partial Y)$ a 1-connected $G$-map of degree one, and $R$ a ring such that $\mathbb{Z} \subset R \subset \mathbb{Q}$. In this section we choose solid tori $\left(\cong S^{1} \times D^{2}\right)$ in $X$ and disks in $Y$ so that after $G$-homotopically deforming $f$, it becomes a prenormal map over $R$ in the sense of [7, Definition 7.1].

First we note that the orientation homomorphism $G \rightarrow\{ \pm 1\}$ induced by the $G$-action on $X$ coincides with that for $Y$, namely $w_{X}=w_{Y}$.

Fix a point $y_{0}$ in $\operatorname{Int}\left(Y_{r(G)}\right)$, where

$$
Y_{r(G)}=Y \backslash Y_{s(G)} .
$$

After $G$-homotopically deforming $f$ if necessary, we may assume that $f$ is transverse regular to the point $y_{0}$. We can choose a tiny disk neighborhood $D_{Y}^{3}$ of $y_{0}$ in $\operatorname{Int}\left(Y_{r(G)}\right)$ and tiny disk neighborhoods $D_{z}$ of $z \in f^{-1}\left(y_{0}\right) \operatorname{in} \operatorname{Int}\left(X_{r(G)}\right)$ such that $D_{Y}^{3} \cap g D_{Y}^{3}=\emptyset$ if $g \neq e(g \in G), D_{z_{1}} \cap g D_{z_{2}}=\emptyset$ if $z_{1} \neq z_{2}$ or $g \neq e$ $\left(z_{1}, z_{2} \in f^{-1}\left(y_{0}\right), g \in G\right)$, and

$$
f^{-1}\left(\operatorname{Int}\left(D_{Y}\right)\right)=\coprod_{z \in f^{-1}\left(y_{0}\right)} \operatorname{Int}\left(D_{z}\right) .
$$

Fix a reference point $y_{1}$ in $\partial D_{Y}^{3}$. Arbitrarily choose and fix $z_{1} \in f^{-1}\left(y_{0}\right)$ and take connecting tubes $\left(\cong I \times D^{2}\right)$ between $\partial D_{z_{1}}$ and the other $\partial D_{z}(z \in$ $\left.f^{-1}\left(y_{0}\right) \backslash\left\{z_{1}\right\}\right)$ in general position of

$$
\operatorname{Int}\left(X_{r(G)}\right) \backslash \bigcup_{w \in f^{-1}\left(y_{0}\right)}\left(G \operatorname{Int}\left(D_{w}\right)\right) .
$$

Since $\pi_{1}(f): \pi_{1}(X) \rightarrow \pi_{1}(Y)$ is surjective, we can choose the connecting tubes so that $f$ maps them to $\left\{y_{1}\right\}$ after $G$-homotopical deformation of $f$. Let $D_{X}^{3}$ be the union of all $D_{z}$ and the connecting tubes. Smoothing corners, $D_{X}^{3}$ becomes diffeomorphic to a 3-dimensional disk. Moreover it holds that $D_{X}^{3} \cap g D_{X}^{3}=\emptyset$ if $g \neq e(g \in G)$, and $f\left(D_{X}^{3}\right)=D_{Y}^{3}$.

Next take solid tori $T_{1}, \ldots, T_{m}\left(\cong S^{1} \times D^{2}\right)$ in general position in

$$
\operatorname{Int}\left(X_{r(G)} \backslash G D_{X}^{3}\right)
$$

such that their cores $\left(\cong S^{1}\right)$ generate $\operatorname{Ker}\left(\pi_{1}(f)\right) \otimes_{\mathbb{Z}} R$. Let $\beta_{j}: S^{1} \times D^{2} \rightarrow T_{j}$ be orientation preserving diffeomorphisms and let $e_{j}$ and $f_{j}$ be the meridians and longitudes of $\beta_{j}$ respectively. Take a reference point $x_{1} \in \partial D_{X}^{3}$ and a tiny 
disk neighborhood $D_{X}^{2}$ of $x_{1}$ in $\partial D_{X}^{3}$. Take connecting tubes between $D_{X}^{2}$ and $\partial T_{j}$ in general position of

$$
\operatorname{Int}\left(X_{r(G)}\right) \backslash \operatorname{Int}\left(G D_{X}^{3}\right) \backslash \coprod_{j=1}^{m} \operatorname{Int}\left(G T_{j}\right) .
$$

We may suppose that $f$ maps all $T_{j}$ and connecting tubes to $\left\{y_{1}\right\}$. Let $T(m)$ be the union of $D_{X}^{3}, T_{1}, \ldots, T_{m}$ and the connecting tubes. Then $T(m)$ is a solid torus of genus $m$. We may suppose that all $e_{j}$ and $f_{j}$ lie on $\partial T(m)$.

We define

$$
U=G T(m), \quad V=G D_{Y}^{3}, \quad X_{0}=X \backslash \operatorname{Int}(U) \text { and } Y_{0}=Y \backslash \operatorname{Int}(V) .
$$

By (1.1.1), $\partial f: \partial X \rightarrow \partial Y$ is not only an $R$-homology equivalence but also an $R\left[\pi_{1}(Y)\right]$-homology equivalence. Since $f$ is a map of degree one, $f$ is a prenormal map over $R$ as well as over $R\left[\pi_{1}(Y)\right]$ in the sense of [7, Definition 7.1].

Let $\Lambda$ denote $R$ or $R\left[\pi_{1}(Y)\right]$. If $\partial f: \partial X \rightarrow \partial Y$ is a $\Lambda$-homology equivalence then we obtain the associated butterfly diagram over $\Lambda$.

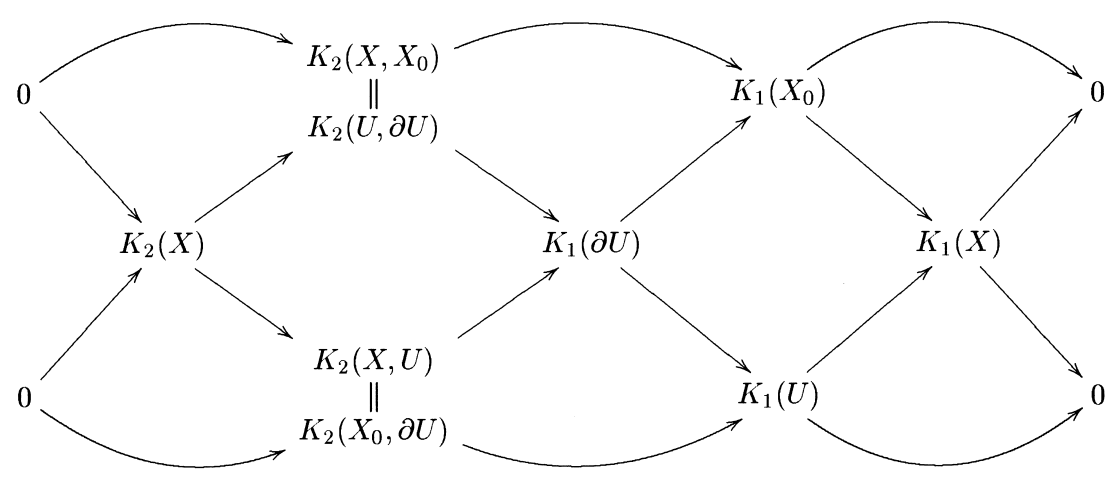

Diagram 3.1

We note that $K_{1}(\partial U ; \Lambda)$ and $K_{2}(U, \partial U ; \Lambda)$ are $\Lambda$-free modules of rank $2 m$ and $m$ respectively. If (1.1.2) is fulfilled then $K_{1}\left(X_{0} ; R\right)$ and $K_{2}(X, U ; R)$ are stably free $R[G]$-modules. In such a case, taking sufficiently large $m$, we may assume that these two modules are free $R[G]$-modules. Set

$$
\begin{aligned}
& G(2)_{+}=\left\{g \in G(2) \mid w_{Y}(g)=1\right\} \text { and } \\
& G(2)_{-}=\left\{g \in G(2) \mid w_{Y}(g)=-1\right\} .
\end{aligned}
$$


We adopt -1 as the symmetry of $R[G]$. Let $Q$ be a subset of $\{e\} \cup G(2)_{+}$and $\Gamma=\Gamma Q$ the smallest form parameter that includes $Q$. Using the $R[G]$-basis

$$
\left\{e_{1}, \ldots, e_{m}, f_{1}, \ldots, f_{m}\right\}
$$

of $K=K_{1}(\partial U ; R)$, we algebraically define the sesquilinear form $B: K \times K \rightarrow$ $R[G]$ over the ring $R[G]$ and the $\Gamma$-quadratic form $q: K \rightarrow R[G] / \Gamma$ by

$$
\begin{aligned}
B\left(\sum_{i}\left(a_{i} e_{i}+b_{i} f_{i}\right), \sum_{j}\left(c_{j} e_{j}+d_{j} f_{j}\right)\right) & =\left(\sum_{i} d_{i} \overline{a_{i}}-c_{i} \overline{b_{i}}\right) \text { and } \\
q\left(\sum_{i}\left(a_{i} e_{i}+b_{i} f_{i}\right)\right) & =\sum_{i}\left[b_{i} \overline{a_{i}}\right]
\end{aligned}
$$

where $a_{i}, b_{i}, c_{j}$ and $d_{j}$ are elements in $R[G]$. Then, $B$ coincides with the geometric equivariant intersection form on $K$. Thus,

$$
B\left(K_{2}(X, U ; R), K_{2}(X, U ; R)\right)=\{0\} .
$$

If an automorphism $\alpha$ on the quadratic module $\left(K_{1}(\partial U ; R), B, q\right)$ satisfies

$$
\alpha\left(K_{2}(U, \partial U ; R)\right)=K_{2}\left(X_{0}, \partial U ; R\right)
$$

then $\alpha$ is said to be preferable. For a preferable automorphism $\alpha$, the matrix $\operatorname{Mat}(\alpha)$ associated with $\alpha$ with respect to the basis $\left\{e_{1}, \ldots, e_{m}, f_{1}, \ldots, f_{m}\right\}$ is called a surgery matrix. In the case where a preferable automorphism exists, the based quadratic module $\left(K_{1}(\partial U ; R), B, q\right)$ determines the surgery matrix uniquely up to the left action of $\mathrm{TU}_{m}$.

Decompose $G$ into the disjoint union of the form

$$
G=\{e\} \amalg G(2) \amalg C \amalg C^{-1},
$$

where $C^{-1}=\left\{g^{-1} \mid g \in C\right\}$. Then, the map $q$ can be regarded as the collection of maps $q_{g}$, where $g \in(\{e\} \cup G(2) \cup C) \backslash Q$, such that

$$
\begin{aligned}
& q_{g}: K \rightarrow R / 2 R \quad\left(g \in\{e\} \cup G(2)_{+} \backslash Q\right), \\
& q_{g}: K \rightarrow R \quad\left(g \in G(2)_{-}\right) \text {and } \\
& q_{g}: K \rightarrow R \quad(g \in C),
\end{aligned}
$$

via the relation

$$
q(x)=\sum_{g \in(\{e\} \cup G(2) \cup C) \backslash Q}\left[q_{g}(x) g\right] \quad(x \in K) .
$$


Note that

$$
B(x, x)=\widetilde{q(x)}-\widetilde{\widetilde{q(x)}}
$$

where $\widetilde{q(x)}$ is a lifting of $q(x)$. Thus, it follows from (3.2) that

$$
q_{g}\left(K_{2}(X, U ; R)\right)=\{0\} \quad\left(g \in G(2)_{-} \cup C\right),
$$

and we conclude

Lemma 3.4. Let $f:(X, \partial X) \rightarrow(Y, \partial Y)$ be as in Theorem 1.3. Then, after a G-homotopical deformation of $(f, b)$, one obtains Diagram 3.1 and the quadratic module $(K, B, q)$ over $R[G]$, where $K=K_{1}(\partial U ; R), B: K \times K \rightarrow$ $R[G]$ and $q: K \rightarrow R[G] / \max$, for which $K_{2}(X, U ; R)$ is totally isotropic.

Definition 3.5. Let $f:(X, \partial X) \rightarrow(Y, \partial Y)$ be as in Theorem 1.3. We define $\sigma(f)$ by

$$
\sigma(f)=[\operatorname{Mat}(\alpha)] \in W_{3}(R[G], \max ; \text { triv }),
$$

after choosing an arbitrary preferable automorphism $\alpha$ of the quadratic module $(K, B, q)$ over $R[G]$, where $K=K_{1}(\partial U ; R), B: K \times K \rightarrow R[G]$ and $q: K \rightarrow$ $R[G] / \max$.

Remark 3.6. The algebraic element $\sigma(f)$ above is not necessarily uniquely determined by the originally given $G$-map $f$. If the reader likes to obtain a unique algebraic object, then he can adopt

$$
\begin{aligned}
& \boldsymbol{\sigma}(f)=\left\{\sigma\left(f^{\prime}\right) \in W_{3}(R[G], \max ; \text { triv })\right. \\
& \text { possible }\left\{T_{1}, \ldots, T_{m}\right\},\left\{\beta_{1}, \ldots, \beta_{m}\right\} \text {, and } f^{\prime} \text { which } \\
& \text { is } G \text {-homotopic to } f \text { and for which } \sigma\left(f^{\prime}\right) \text { can be } \\
& \text { defined with respect to }\left\{\beta_{1}, \ldots, \beta_{m}\right\}
\end{aligned}
$$

instead and read the condition $\sigma(f)=0$ in Theorem 1.3 as $\boldsymbol{\sigma}(f) \ni 0$.

In the remainder of the current section, we discuss the triviality of $q_{g}$ for

$$
g \in\{e\} \cup G(2)_{+} \backslash G(Y)
$$

Let $\eta_{+}$and $\eta_{-}$be oriented real $G$-vector bundles over $Y$ such that $\eta_{+} \supset$ $\varepsilon_{Y}\left(\mathbb{R}^{4}\right)$. Let $b: T(X) \oplus f^{*} \eta_{-} \rightarrow \eta_{+}$be an orientation preserving map of $G$ vector bundles covering $f: X \rightarrow Y$. Let $\boldsymbol{\omega}_{+}$and $\boldsymbol{\omega}_{-}$be $G$-frames over $V$ of 
the oriented $G$-vector bundles $\eta_{+}$and $\eta_{-}$respectively. Let $b_{U}^{*} \boldsymbol{\omega}_{+}$denote the $G$-frame over $U$ induced by

$$
b_{U}=b \mid: T(X)_{U} \oplus\left(f^{*} \eta_{-}\right)_{U} \rightarrow\left(\eta_{+}\right)_{V}
$$

from $\boldsymbol{\omega}_{+}$, and let $\left.f\right|_{U} ^{*} \boldsymbol{\omega}_{-}$denote the $G$-frame over $U$ of $f^{*} \eta_{-}$induced by the canonical map

$$
\left.f\right|_{U} ^{*} \eta_{-} \rightarrow\left(\eta_{-}\right)_{V}
$$

covering $\left.f\right|_{U}$ from $\boldsymbol{\omega}_{-}$. Then, applying [7, Proposition 2.2] to $X$ replaced by $T(m)$ above, we obtain a $G$-frame $\boldsymbol{\kappa}$ over $U$ of the oriented $G$-vector bundle $T(U)$ such that $\boldsymbol{\kappa}+\left.f\right|_{U} ^{*} \boldsymbol{\omega}_{-}$is homotopic to $b_{U}^{*} \boldsymbol{\omega}_{+}$.

Hypothesis 3.7. In the case with bundle data as above, we assume that all $\beta_{1}, \ldots, \beta_{m}$ are preferable in the sense of [7, Definition 7.4].

If $\beta_{j}: S^{1} \times D^{2} \rightarrow T_{j}$ is not preferable then we can replace $\beta_{j}$ by a preferable one. For example, adopt $\beta_{j}^{\prime}: S^{1} \times D^{2} \rightarrow T_{j}$ defined by

$$
\beta_{j}^{\prime}\left(z_{1}, z_{2}\right)=\beta_{j}\left(z_{1}, z_{1} z_{2}\right) \quad\left(z_{1}, z_{2} \in \mathbb{C} \text { with }\left|z_{1}\right|=1 \text { and }\left|z_{2}\right| \leq 1\right)
$$

instead of $\beta_{j}$. Thus, the arguments developped so far do not lose generality by the hypothesis.

By [7, Theorem 8.1], the quadratic form $q_{e}: K_{1}(\partial U ; R) \rightarrow R / 2 R$ vanishes on $K_{2}(X, U ; R)$.

Let $g$ be an element in $G(2)_{+} \backslash G(Y)$. Then, $g$ acts freely on $X$ and $Y$. Thus, $X /\langle g\rangle$ and $Y /\langle g\rangle$ are oriented manifolds and the induced map

$$
f /\langle g\rangle:(X /\langle g\rangle, \partial X /\langle g\rangle) \rightarrow(Y /\langle g\rangle, \partial Y /\langle g\rangle)
$$

is a map of degree one.

Lemma 3.8. For each $g \in G(2)_{+} \backslash G(Y)$, one has $q_{g}\left(K_{2}(X, U ; R)\right)=$ $\{0\}$.

Proof. For the proof, by the definition of $q$, we may suppose

$$
G=\langle g\rangle
$$

Note that

$$
\varepsilon(q(x))=\varepsilon\left(q_{e}(x)+q_{g}(x) g\right)=q_{e}(x)+q_{g}(x),
$$


where $\varepsilon:(R / 2 R)[G] \rightarrow R / 2 R$ is the augmentation homomorphism. If $x \in$ $K_{2}(X, U ; R)$ then $q_{e}(x)=0$ and hence $\varepsilon(q(x))=q_{g}(x)$. But $\varepsilon \circ q$ coincides with $q^{\prime} \circ \pi$, where $q^{\prime}: K_{1}(\partial U / G ; R) \rightarrow R / 2 R$ is the algebraic quadratic form associated with the compositions of $\beta_{i}, i=1, \ldots, m$, with the projection map $X \rightarrow X / G$, and $\pi: K_{1}(\partial U ; R) \rightarrow K_{1}(\partial U / G ; R)$ is the canonical homomorphism. By [7, Theorem 8.1], $q^{\prime}(z)=0$ holds for any $z \in K_{2}(X / G, U / G ; R)$. Thus we obtain

$$
q_{g}(x)=\varepsilon(q(x))=q^{\prime}(\pi(x))=0
$$

for $x \in K_{2}(X, U ; R)$.

Q.E.D.

Putting all together, we obtain

Lemma 3.9. Let $(f, b)$ be as in Theorem 1.1. Then, after a G-homotopical deformation of $(f, b)$, one obtains Diagram 3.1 and the quadratic module $(K, B, q)$ over $R[G]$, where $K=K_{1}(\partial U ; R), B: K \times K \rightarrow R[G]$ and $q: K \rightarrow R[G] / \Gamma G(Y)$, for which $K_{2}(X, U ; R)$ is totally isotropic.

Definition 3.10. $\quad$ Let $(f, b)$ be as in Theorem 1.1. We define $\sigma(f, b)$ by

$$
\sigma(f, b)=[\operatorname{Mat}(\alpha)] \in W_{3}(R[G], \Gamma G(Y) ; w),
$$

after choosing an arbitrary preferable automorphism $\alpha$ on the quadratic module $(K, B, q)$ over $R[G]$, where $K=K_{1}(\partial U ; R), B: K \times K \rightarrow R[G]$ and $q: K \rightarrow$ $R[G] / \Gamma G(Y)$.

\section{$\S 4$. Proof of Theorems 1.1 and $\mathbf{1 . 3}$}

Let $(f, b)$ be as in Theorem 1.1 and $\left(K_{1}(\partial U ; R), B, q\right)$ as in Definition 3.10.

$\boldsymbol{G}$-surgery along $\boldsymbol{\beta}_{\boldsymbol{k}}$. We observe how the surgery matrix $\operatorname{Mat}(\alpha)$ in Definition 3.10 is influenced by the $G$-surgery along $\beta_{k}: S^{1} \times D^{2} \rightarrow X$, where $k$ is a fixed integer with $1 \leq k \leq m$. Let $f^{\prime}: X^{\prime} \rightarrow Y$ be the $G$-map resulting from the $G$-surgery along $\beta_{k}$, let $\beta_{i}^{\prime}=\beta_{i}$ for all $i \neq k$ and let $\beta_{k}^{\prime}$ be the dual to $\beta_{k}$. Since $\partial \operatorname{Im}\left(\beta_{i}^{\prime}\right)=\partial \operatorname{Im}\left(\beta_{i}\right)$ we can use the same connecting tubes. We obtain $X_{0}^{\prime}, T(m)^{\prime}$ and $U^{\prime}$ for $\left\{\beta_{i}^{\prime}\right\}$ instead of $X_{0}, T(m)$ and $U$ obtained for $\left\{\beta_{i}\right\}$, respectively. The meridian and longitude of $\beta_{i}^{\prime}$ are denoted by $e_{i}^{\prime}$ and $f_{i}^{\prime}$ respectively for each $i$. Clearly, $X_{0}^{\prime}=X_{0}$ and $\partial U^{\prime}=\partial U$. However the new 
basis of $K_{1}\left(\partial U^{\prime} ; R\right)$ is $\left\{e_{1}^{\prime}, \ldots, e_{m}^{\prime}, f_{1}^{\prime}, \ldots, f_{m}^{\prime}\right\}$. Define an $R[G]$-endomorphism $\alpha^{\prime}$ of $K_{1}\left(\partial G U^{\prime} ; R\right)$ by

$$
\alpha^{\prime}\left(\begin{array}{c}
e_{1}^{\prime} \\
\vdots \\
e_{m}^{\prime} \\
f_{1}^{\prime} \\
\vdots \\
f_{m}^{\prime}
\end{array}\right)=\alpha\left(\begin{array}{c}
e_{1} \\
\vdots \\
e_{m} \\
f_{1} \\
\vdots \\
f_{m}
\end{array}\right)
$$

Then, noting that $e_{k}=f_{k}^{\prime}$ and $f_{k}=-e_{k}^{\prime}$, we obtain

$$
\alpha^{\prime}\left(\begin{array}{c}
e_{1}^{\prime} \\
\vdots \\
e_{m}^{\prime} \\
f_{1}^{\prime} \\
\vdots \\
f_{m}^{\prime}
\end{array}\right)=\operatorname{Mat}(\alpha)\left(\begin{array}{c}
e_{1} \\
\vdots \\
e_{m} \\
f_{1} \\
\vdots \\
f_{m}
\end{array}\right)=\operatorname{Mat}(\alpha) \sigma_{k}\left(\begin{array}{c}
e_{1}^{\prime} \\
\vdots \\
e_{m}^{\prime} \\
f_{1}^{\prime} \\
\vdots \\
f_{m}^{\prime}
\end{array}\right) .
$$

Thus, $\alpha^{\prime}$ is an automorphism of $\left(K_{1}\left(\partial U^{\prime} ; R\right), B^{\prime}, q^{\prime}\right)$, where $B^{\prime}=B$ and $q^{\prime}=q$. Furthermore it holds that

$$
\begin{aligned}
\alpha^{\prime}\left(K_{2}\left(U^{\prime}, \partial U^{\prime} ; R\right)\right) & =\left\langle\alpha^{\prime}\left(e_{1}^{\prime}\right), \ldots, \alpha^{\prime}\left(e_{m}^{\prime}\right)\right\rangle_{R[G]}=\left\langle\alpha\left(e_{1}\right), \ldots, \alpha\left(e_{m}\right)\right\rangle_{R[G]} \\
& =K_{2}\left(X_{0}, \partial U ; R\right)=K_{2}\left(X_{0}^{\prime}, \partial U^{\prime} ; R\right) .
\end{aligned}
$$

This shows that $\alpha^{\prime}$ is preferable for defining $\sigma\left(f^{\prime}, b^{\prime}\right)$. By definition, the surgery matrix $\operatorname{Mat}\left(\alpha^{\prime}\right)$ associated with $\alpha^{\prime}$ is $\operatorname{Mat}(\alpha) \sigma_{k}$. We have proved

Proposition 4.1. Let $\operatorname{Mat}(\alpha)$ be a surgery matrix for $\left\{\beta_{1}, \ldots, \beta_{m}\right\}$. Then the $G$-surgery along $\beta_{k}$ alters the matrix $\operatorname{Mat}(\alpha)$ to $\operatorname{Mat}(\alpha) \sigma_{k}$.

Reversing $\boldsymbol{\beta}_{\boldsymbol{k}}$. Let $k$ be an integer with $1 \leq k \leq m$. We observe how the surgery matrix $\operatorname{Mat}(\alpha)$ in Definition 3.10 is influenced by replacing $\beta_{k}$ with $\beta_{k}^{\prime}: S^{1} \times D^{2} \rightarrow T_{j}$ defined by

$$
\beta_{k}^{\prime}(x, y)=\beta(\bar{x}, \bar{y}) \text { for } x \in S^{1} \text { and } y \in D^{2},
$$

where $S^{1}$ and $D^{2}$ are regarded as the unit circle and disk of $\mathbb{C}$ respectively, and $\bar{x}$ and $\bar{y}$ stand for the complex conjugates of $x$ and $y$ respectively. For $i \neq k$, we set $\beta_{i}^{\prime}=\beta_{i}$. Moreover, all new data derived from $\left\{\beta_{1}^{\prime}, \ldots, \beta_{m}^{\prime}\right\}$ are denoted here by the initial notation with prime ', e.g. $e_{i}^{\prime}, f_{i}^{\prime}, U^{\prime}$ etc. Then, 
we may adopt $U^{\prime}=U$ in order to obtain a surgery matrix for $\left\{\beta_{1}^{\prime}, \ldots, \beta_{m}^{\prime}\right\}$. Obviously, $e_{k}^{\prime}=-e_{k}, f_{k}^{\prime}=-f_{k}, e_{j}^{\prime}=e_{j}$ and $f_{j}^{\prime}=f_{j}, j \neq k$, hold. Define $\alpha^{\prime}: K_{1}\left(\partial G U^{\prime} ; R\right) \rightarrow K_{1}\left(\partial G U^{\prime} ; R\right)$ by

$$
\alpha^{\prime}\left(\begin{array}{c}
e_{1}^{\prime} \\
\vdots \\
e_{k}^{\prime} \\
\vdots \\
e_{m}^{\prime} \\
f_{1}^{\prime} \\
\vdots \\
f_{k}^{\prime} \\
\vdots \\
f_{m}^{\prime}
\end{array}\right)=\alpha\left(\begin{array}{c}
e_{1} \\
\vdots \\
e_{k} \\
\vdots \\
e_{m} \\
f_{1} \\
\vdots \\
f_{k} \\
\vdots \\
f_{m}
\end{array}\right) .
$$

This $\alpha^{\prime}$ is a preferable automorphism, i.e. $\alpha^{\prime}\left(K_{2}\left(U^{\prime}, \partial U^{\prime} ; R\right)\right)=K_{2}\left(X_{0}^{\prime}, \partial U^{\prime} ; R\right)$. It is easy to see that the associated surgery matrix $\operatorname{Mat}\left(\alpha^{\prime}\right)$ is $\operatorname{Mat}(\alpha) \sigma_{k}^{2}$. Thus we obtain

Proposition 4.2. Let $\operatorname{Mat}(\alpha)$ be a surgery matrix with respect to $\left\{\beta_{1}, \ldots, \beta_{m}\right\}$. Then reversing $\beta_{k}$ alters the matrix $\operatorname{Mat}(\alpha)$ to $\operatorname{Mat}(\alpha) \sigma_{k}^{2}$.

For $a \in R^{\times}$we define a $2 \times 2$-matrix $\iota(a)$ by

$$
\iota(a)=\left(\begin{array}{cc}
a & 0 \\
0 & 1 / a
\end{array}\right) .
$$

For an integer $k$ with $1 \leq k \leq m$ we define a $2 m \times 2 m$-matrix $\iota_{k}(a)$ by

$$
\iota_{k}(a)=I_{2 k-2} \oplus \iota(a) \oplus I_{2 m-2 k} .
$$

Multiplication of $\boldsymbol{\beta}_{\boldsymbol{k}}$. Let $a$ be a natural number such that $1 / a \in R$. Then, we can take an orientation preserving embedding $\beta_{k}^{\prime}: S^{1} \times D^{2} \rightarrow \operatorname{Int}\left(T_{k}\right)$, where $T_{k}=\operatorname{Im}\left(\beta_{k}\right)$, such that $e_{k}^{\prime}=(1 / a) e_{k}$ and $f_{k}^{\prime}=a f_{k}$ in $H_{1}\left(T_{k} \backslash \operatorname{Int}\left(T_{k}^{\prime}\right) ; R\right)$, where $T_{k}^{\prime}=\operatorname{Im}\left(\beta_{k}^{\prime}\right)$. We call $\beta_{k}^{\prime}$ an a-multiplication of $\beta_{k}$. For $i \neq k$, we adopt $\beta_{i}^{\prime}=\beta_{i}$. Then, subsets $U^{\prime}$ and $X_{0}^{\prime}$ of $X$ are obtained from $\left\{\beta_{1}^{\prime}, \ldots, \beta_{m}^{\prime}\right\}$. Observe the canonical exact sequence

$$
K_{2}\left(U, U^{\prime} ; R\right) \rightarrow K_{2}\left(X, U^{\prime} ; R\right) \rightarrow K_{2}(X, U ; R) \rightarrow K_{1}\left(U, U^{\prime} ; R\right) .
$$


Since $K_{2}\left(U, U^{\prime} ; R\right)=0$ and $K_{1}\left(U, U^{\prime} ; R\right)=0, K_{2}\left(X, U^{\prime} ; R\right)=K_{2}(X, U ; R)$ holds via the canonical map. Let us observe how replacing $\beta_{k}$ with $\beta_{k}^{\prime}$ influences the surgery matrix Mat $(\alpha)$. Formally using the identities $e_{i}=e_{i}^{\prime}$ and $f_{i}=f_{i}^{\prime}$ $(i \neq k)$ together with $e_{k}=a e_{k}^{\prime}$ and $f_{k}=(1 / a) f_{k}^{\prime}$, we define $\alpha^{\prime}: K_{1}\left(\partial U^{\prime} ; R\right) \rightarrow$ $K_{1}\left(\partial U^{\prime} ; R\right)$ by

$$
\alpha^{\prime}\left(\begin{array}{c}
e_{1}^{\prime} \\
\vdots \\
e_{k}^{\prime} \\
\vdots \\
e_{m}^{\prime} \\
f_{1}^{\prime} \\
\vdots \\
f_{k}^{\prime} \\
\vdots \\
f_{m}^{\prime}
\end{array}\right)=\alpha\left(\begin{array}{c}
e_{1} \\
\vdots \\
e_{k} \\
\vdots \\
e_{m} \\
f_{1} \\
\vdots \\
f_{k} \\
\vdots \\
f_{m}
\end{array}\right) .
$$

(The elements $\alpha\left(e_{i}\right)$ and $\alpha\left(f_{i}\right)$ of the right hand side are linear combinations of $e_{s}$ and $f_{t}, 1 \leq s, t \leq m$. Thus they are linear combinations of $e_{s}^{\prime}$ and $f_{t}^{\prime}$.) It holds that $\alpha^{\prime}\left(K_{2}\left(U^{\prime}, \partial U^{\prime} ; R\right)\right)=K_{2}\left(X_{0}^{\prime}, \partial U^{\prime} ; R\right)$, namely $\alpha^{\prime}$ is preferable. The matrix $\operatorname{Mat}\left(\alpha^{\prime}\right)$ associated with $\alpha^{\prime}$ with respect to $\left\{e_{1}^{\prime}, \ldots, e_{m}^{\prime}, f_{1}^{\prime}, \ldots, f_{m}^{\prime}\right\}$ is $\operatorname{Mat}(\alpha) \iota_{k}(a)$. This concludes

Proposition 4.3. Let $\operatorname{Mat}(\alpha)$ be a surgery matrix with respect to $\left\{\beta_{1}, \ldots, \beta_{m}\right\}$ and a natural number invertible in $R$. Then taking a-multiplication of $\beta_{k}$ converts the matrix $\operatorname{Mat}(\alpha)$ to $\operatorname{Mat}(\alpha) \iota_{k}(a)$.

Stabilization. We discuss a stabilization of the surgery matrix $\operatorname{Mat}(\alpha)$.

Proposition 4.4. Let $\operatorname{Mat}(\alpha)$ be a surgery matrix with respect to $\left\{\beta_{1}, \ldots, \beta_{m}\right\}$. Let $\beta_{m+1}: S^{1} \times D^{2} \rightarrow X$ be an additional orientation preserving trivial embedding. Perform the $G$-surgery along $\beta_{m+1}$, and let $\beta_{m+1}^{\prime}$ : $S^{1} \times D^{2} \rightarrow X^{\prime}$ be the dual to $\beta_{m+1}$. Furthermore let $\beta_{m+1}^{\prime \prime}: S^{1} \times D^{2} \rightarrow$ $X^{\prime}$ be the embedding defined by $\beta^{\prime \prime}(x, y)=\beta^{\prime}(\bar{x}, \bar{y})$ for $x \in S^{1}$ and $y \in$ $D^{2}$. Then $j_{m, m+1}(\operatorname{Mat}(\alpha))\left(=\operatorname{Mat}(\alpha) \oplus I_{2}\right)$ is a new surgery matrix for $\left\{\beta_{1}, \ldots, \beta_{m}, \beta_{m+1}^{\prime \prime}\right\}$.

Proof. The matrix Mat $(\alpha) \oplus \sigma$ is a surgery matrix for $\left\{\beta_{1}, \ldots, \beta_{m}, \beta_{m+1}\right\}$. By Proposition 4.1, $(\operatorname{Mat}(\alpha) \oplus \sigma) \sigma_{m+1}$ is a surgery matrix with respect to 
$\left\{\beta_{1}, \ldots, \beta_{m}, \beta_{m+1}^{\prime}\right\}$. Clearly,

$$
(\operatorname{Mat}(\alpha) \oplus \sigma) \sigma_{m+1}=\operatorname{Mat}(\alpha) \oplus\left(-I_{2}\right) .
$$

Thus, by Proposition 4.2, $\operatorname{Mat}(\alpha) \oplus I_{2}$ is a surgery matrix with respect to $\left\{\beta_{1}, \ldots, \beta_{m}, \beta_{m+1}^{\prime \prime}\right\}$.

Q.E.D.

This proposition allows us to treat surgery matrices in stable range.

Let $\Sigma_{m}=\sigma \oplus \sigma \oplus \ldots \oplus \sigma$ (m-fold sum). By definition, $\Sigma_{m}$ lies in $\mathrm{FU}_{m}(R[G], \Gamma G(Y))$. It is remarkable that

$$
\begin{aligned}
\sigma(f)=0 & \Rightarrow \operatorname{Mat}(\alpha) \in \mathrm{RU}_{m} \quad(\text { for large } m) \\
& \Rightarrow \operatorname{Mat}(\alpha) \in \mathrm{TU}_{m} \Sigma_{m} \mathrm{FU}_{m} \\
& \Rightarrow \operatorname{TU}_{m} \operatorname{Mat}(\alpha) F_{1} \ldots F_{\ell} \ni \Sigma_{m} \text { for some } F_{1}, \ldots, F_{\ell} \in \mathrm{FU}_{m} .
\end{aligned}
$$

Here we may suppose that each $F_{i}$ above has one of the forms in the list

$$
\begin{cases}(4.5 .1) & \varepsilon_{m+k, k}(g+\bar{g}), \quad \varepsilon_{m+k, k}(-(g+\bar{g})) \quad(g \in G), \\ (4.5 .2) & \varepsilon_{m+h, k}(g), \quad \varepsilon_{m+h, k}(-g) \quad(g \in G \text { and } h \neq k), \\ (4.5 .3) & \varepsilon_{m+k, k}(g), \quad \varepsilon_{m+k, k}(-g) \quad(g \in G(Y)) \\ (4.5 .4) & \iota_{k}(a), \quad \iota_{k}(-a) \quad(a \in \mathbb{N} \text { with } 1 / a \in R), \\ (4.5 .5) & \sigma_{k},\end{cases}
$$

where $1 \leq h, k \leq m$.

Lemma 4.6. If $\mathrm{TU}_{m} \operatorname{Mat}(\alpha)$ contains $\Sigma_{m}$ then

$$
K_{1}(\partial U ; R)=K_{2}(U, \partial U ; R)+K_{2}\left(X_{0}, \partial U ; R\right),
$$

and $f: X \rightarrow Y$ is an R-homology equivalence.

Proof. This follows from Diagram 3.1.

Q.E.D.

For proving Theorem 1.1, it suffices, for each matrix $F$ in (4.5), to find an operation which alters a surgery matrix $\operatorname{Mat}(\alpha)$ to $\operatorname{Mat}(\alpha) F$. We have already found operations for $F$ of types (4.5.4) and (4.5.5). Thus, it suffices to prove

Proposition 4.7. There exists a G-surgery operation which alters the surgery matrix $\operatorname{Mat}(\alpha)$ to $\operatorname{Mat}(\alpha) F$ for each matrix

$$
\begin{aligned}
& F=\varepsilon_{m+k, k}( \pm(g+\bar{g})) \quad(g \in G), \\
& F=\varepsilon_{m+h, k}( \pm g) \quad(g \in G \text { and } h \neq k), \\
& F=\varepsilon_{m+k, k}( \pm g) \quad(g \in G(Y)) .
\end{aligned}
$$


Lemma 4.8 (cf. (4.7.1)). Let $g \in G$ and $\epsilon=1$ (resp. -1). Perform an isotopical deformation in $X_{r(G)}$ of a small portion of $\beta_{k}$ and link it to $g \beta_{k}$ in the negative (resp. positive) direction to ge ${ }_{k}$. This alters the surgery matrix $\operatorname{Mat}(\alpha)$ with respect to $\left\{\beta_{1}, \ldots, \beta_{m}\right\}$ to a surgery matrix $\operatorname{Mat}\left(\alpha^{\prime}\right)$ for $\left\{\beta_{1}^{\prime}, \ldots, \beta_{m}^{\prime}\right\}$ such that $\operatorname{Mat}\left(\alpha^{\prime}\right)=\operatorname{Mat}(\alpha) \varepsilon_{m+k, k}(g+\bar{g})\left(\right.$ resp. Mat $\left.(\alpha) \varepsilon_{m+k, k}(-(g+\bar{g}))\right)$, where $\beta_{i}^{\prime}=\beta_{i}$ if $i \neq k$, and $\beta_{k}^{\prime}$ is the embedding obtained after the deformation of $\beta_{k}$.

The domain of $\beta_{k}$ is $S^{1} \times D^{2}$. A small portion above means the part

$$
(\exp ((\pi-\delta) \sqrt{-1}), \exp ((\pi+\delta) \sqrt{-1})) \times D^{2}
$$

of $S^{1} \times D^{2}$ for small $\delta>0$.

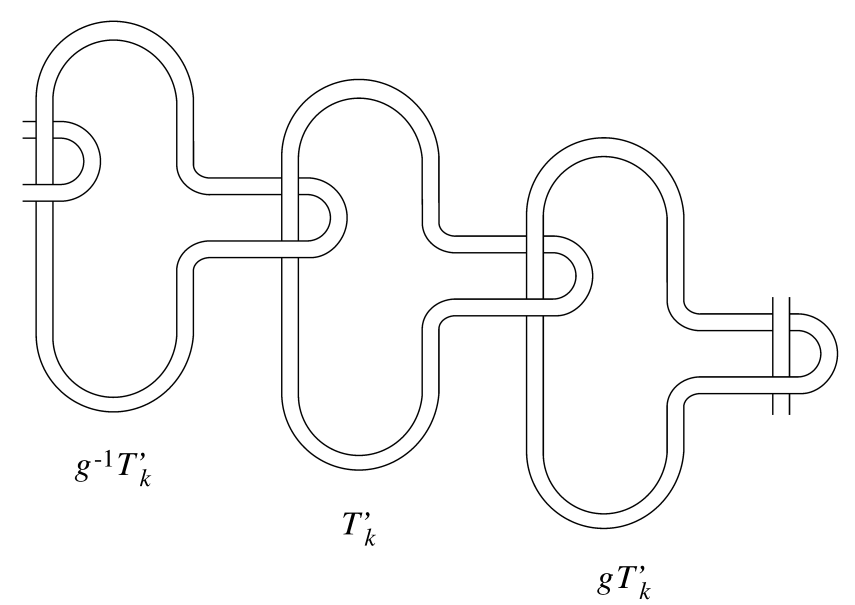

Figure 4.9

Proof. Without loss of generality, we can assume $k=1$. Define an $R[G]$ homomorphism $\beta: K_{1}(\partial G U ; R) \rightarrow K_{1}\left(\partial G U^{\prime} ; R\right)$ by

$$
\left.\beta\left(\begin{array}{c}
e_{1} \\
\vdots \\
e_{m} \\
f_{1} \\
f_{2} \\
\vdots \\
f_{m}
\end{array}\right)=\left(\begin{array}{c}
e_{1}^{\prime} \\
\vdots \\
e_{m}^{\prime} \\
\epsilon(g+\bar{g}) e_{1}^{\prime}+f_{1}^{\prime} \\
f_{2}^{\prime} \\
\vdots \\
f_{m}^{\prime}
\end{array}\right)=\varepsilon_{m+1,1}(\epsilon(g+\bar{g}))\left(\begin{array}{c}
e_{1}^{\prime} \\
\vdots \\
e_{m}^{\prime} \\
f_{1}^{\prime} \\
\vdots \\
f_{m}^{\prime}
\end{array}\right)\right)
$$


Clearly, $\beta$ is an isomorphism $\left(K_{1}(\partial G U ; R), \lambda, \mu\right) \rightarrow\left(K_{1}\left(\partial G U^{\prime} ; R\right), \lambda^{\prime}, \mu^{\prime}\right)$. Moreover it holds that

$$
\beta\left(K_{2}\left(X_{0}, \partial G U ; R\right)\right)=K_{2}\left(X_{0}^{\prime}, \partial G U^{\prime} ; R\right) .
$$

Define an endomorphism $\alpha^{\prime}$ of $K_{1}\left(\partial G U^{\prime} ; R\right)$ by

$$
\alpha^{\prime}\left(\begin{array}{c}
e_{1}^{\prime} \\
\vdots \\
e_{m}^{\prime} \\
f_{1}^{\prime} \\
\vdots \\
f_{m}^{\prime}
\end{array}\right)=\operatorname{Mat}(\alpha) \varepsilon_{m+1,1}(\epsilon(g+\bar{g}))\left(\begin{array}{c}
e_{1}^{\prime} \\
\vdots \\
e_{m}^{\prime} \\
f_{1}^{\prime} \\
\vdots \\
f_{m}^{\prime}
\end{array}\right)
$$

Then $\alpha^{\prime}$ is an automorphism of $\left(K_{1}\left(\partial G U^{\prime} ; R\right), \lambda^{\prime}, \mu^{\prime}\right)$. Since $\alpha^{\prime}\left(e_{i}^{\prime}\right)=\beta \alpha\left(e_{i}\right)$ for all $i=1, \ldots, m$, we have

$$
\alpha^{\prime}\left(K_{2}\left(G U^{\prime}, \partial G U^{\prime} ; R\right)\right)=\beta \alpha\left(K_{2}(G U, \partial G U ; R)\right)=K_{2}\left(X_{0}^{\prime}, \partial G U^{\prime} ; R\right) .
$$

Hence, $\alpha^{\prime}$ is preferable. The associated surgery matrix $\operatorname{Mat}\left(\alpha^{\prime}\right)$ is

$$
\operatorname{Mat}(\alpha) \varepsilon_{m+1,1}(\epsilon(g+\bar{g})) \text {. }
$$

Lemma 4.10 (cf. (4.7.2)). Let $g \in G$ and $\epsilon=1$ (resp. -1). Perform an isotopical deformation in $X_{r(G)}$ of a small portion of $\beta_{h}$ and link it to $g \beta_{k}$ in the negative (resp. positive) direction to ge $e_{k}$. This alters the surgery matrix $\operatorname{Mat}(\alpha)$ for $\left\{\beta_{1}, \ldots, \beta_{m}\right\}$ to a surgery matrix $\operatorname{Mat}\left(\alpha^{\prime}\right)$ for $\left\{\beta_{1}^{\prime}, \ldots, \beta_{m}^{\prime}\right\}$ such that $\operatorname{Mat}\left(\alpha^{\prime}\right)=\operatorname{Mat}(\alpha) \varepsilon_{m+h, k}(g)\left(\right.$ resp. $\left.\operatorname{Mat}(\alpha) \varepsilon_{m+h, k}(-g)\right)$, where $\beta_{i}^{\prime}=\beta_{i}$ if $i \neq h$, and $\beta_{h}^{\prime}$ is the embedding obtained after the deformation of $\beta_{k}$.

Proof. We may suppose that $h=1$ and $k=2$ without loss of generality. Define a $R[G]$-homomorphism $\beta: K_{1}(\partial G U ; R) \rightarrow K_{1}\left(\partial G U^{\prime} ; R\right)$ by

$$
\left.\beta\left(\begin{array}{c}
e_{1} \\
\vdots \\
e_{m} \\
f_{1} \\
f_{2} \\
f_{3} \\
\vdots \\
f_{m}
\end{array}\right)=\left(\begin{array}{c}
e_{1}^{\prime} \\
\vdots \\
e_{m}^{\prime} \\
\epsilon g e_{2}^{\prime}+f_{1}^{\prime} \\
\epsilon \bar{g} e_{1}^{\prime}+f_{2}^{\prime} \\
f_{3}^{\prime} \\
\vdots \\
f_{m}^{\prime}
\end{array}\right)=\varepsilon_{m+1,2}(\epsilon g)\left(\begin{array}{c}
e_{1}^{\prime} \\
\vdots \\
e_{m}^{\prime} \\
f_{1}^{\prime} \\
\vdots \\
f_{m}^{\prime}
\end{array}\right)\right) .
$$


Clearly, $\beta$ is an isomorphism $\left(K_{1}(\partial G U ; R), \lambda, \mu\right) \rightarrow\left(K_{1}\left(\partial G U^{\prime} ; R\right), \lambda^{\prime}, \mu^{\prime}\right)$. It holds that

$$
\beta\left(K_{2}\left(X_{0}, \partial G U ; R\right)\right)=K_{2}\left(X_{0}^{\prime}, \partial G U^{\prime} ; R\right) .
$$

Define an $R[G]$-endomorphism $\alpha^{\prime}$ of $K_{1}\left(\partial G U^{\prime} ; R\right)$ by

$$
\alpha^{\prime}\left(\begin{array}{c}
e_{1}^{\prime} \\
\vdots \\
e_{m}^{\prime} \\
f_{1}^{\prime} \\
\vdots \\
f_{m}^{\prime}
\end{array}\right)=\operatorname{Mat}(\alpha) \varepsilon_{m+1,2}(\epsilon g)\left(\begin{array}{c}
e_{1}^{\prime} \\
\vdots \\
e_{m}^{\prime} \\
f_{1}^{\prime} \\
\vdots \\
f_{m}^{\prime}
\end{array}\right)
$$

Then $\alpha^{\prime}$ is an automorphism of $\left(K_{1}\left(\partial G U^{\prime} ; R\right), \lambda^{\prime}, \mu^{\prime}\right)$. Since $\alpha^{\prime}\left(e_{i}^{\prime}\right)=\beta \alpha\left(e_{i}\right)$ for all $i=1, \ldots, m$, we have

$$
\alpha^{\prime}\left(K_{2}\left(G U^{\prime}, \partial G U^{\prime} ; R\right)\right)=\beta \alpha\left(K_{2}(G U, \partial G U ; R)\right)=K_{2}\left(X_{0}^{\prime}, \partial G U^{\prime} ; R\right)
$$

Hence, $\alpha^{\prime}$ is preferable and the surgery matrix Mat $\left(\alpha^{\prime}\right)$ associated with $\alpha^{\prime}$ is

$$
\operatorname{Mat}(\alpha) \varepsilon_{m+1,2}(\epsilon g)
$$

Next we treat the final case (4.7.3) for $g \in G(Y)$. Choose and fix a connected component $X_{\gamma}^{g}$ of $X^{g}$ such that $\operatorname{dim} X_{\gamma}^{g}=1$. Let $H$ be the principal isotropy subgroup of $G$ on $G X_{\gamma}^{g}$ such that $g \in H$. Set $L=\left(X_{\gamma}^{g}\right)^{H} \backslash\left(X_{\gamma}^{g}\right)^{>H}$. Then, $\operatorname{dim} L=1$, and codim $L=2$. Recall $\operatorname{dim} X^{g^{\prime}} \leq 1$ for all $g^{\prime} \neq e$, because of $(\mathcal{M} 1)$. This implies that $H$ acts freely on the normal fiber of $L$ except the origin. Thus, $H$ must be a cyclic group. We denote a generator of $H$ by $h$. Clearly we get $g=h^{|H| / 2}$. Perform an isotopical deformation of a small portion of $\beta_{k}$ and link it to $L$ and set it again in general position. Then $\beta_{k}$ is linked to $h^{1} \beta_{k}, h^{2} \beta_{k}, \ldots, h^{|H|-1} \beta_{k}$. Consider an element $a=h^{i}$ with $1 \leq i \leq|H| / 2-1$. Since $\operatorname{dim} X^{a}=1$, the action of $a$ on $X$ preserves the orientation and hence $\epsilon(a+\bar{a})=\epsilon\left(a+a^{-1}\right)$. Using the isotopical defomation in Lemma 4.8, we can eliminate the linking of $\beta_{k}$ with $h^{i} \beta_{k}$ and the linking of $\beta_{k}$ with $h^{|H|-i} \beta_{k}$ in a pair. Regarding $\left\{h^{1}, h^{2}, \ldots, h^{|H|-1}\right\}$ as $\left\{\left(h^{1}, h^{|H|-1}\right),\left(h^{2}, h^{|H|-2}\right), \ldots,\left(h^{|H| / 2-1}, h^{|H| / 2+1}\right), h^{|H| / 2}\right\}$, we use the technique of pairwise elimination and remove the linking of $\beta_{k}$ with $h^{i} \beta_{k}$, where $1 \leq i \leq|H|-1$ and $i \neq|H| / 2$. Thus, there exists an isotopical deformation of a small portion of $\beta_{k}$ such that $\beta_{k}$ is linked only to $g \beta_{k}$. 
Lemma 4.11 (cf. (4.7.3)). Let $g \in G(Y)$ and $\epsilon=1$ (resp. -1). Choose and fix a connected component $X_{\gamma}^{g}$ of $X^{g}$ such that $\operatorname{dim} X_{\gamma}^{g}=1$. Perform an isotopical deformation of a small portion of $\beta_{k}$ and link it only to $g \beta_{k}$ in the negative (resp. positive) direction to ge $e_{k}$. This alters the surgery matrix $\operatorname{Mat}(\alpha)$ for $\left\{\beta_{1}, \ldots, \beta_{m}\right\}$ to a surgery matrix $\operatorname{Mat}\left(\alpha^{\prime}\right)$ for $\left\{\beta_{1}^{\prime}, \ldots, \beta_{m}^{\prime}\right\}$ such that $\operatorname{Mat}\left(\alpha^{\prime}\right)=\operatorname{Mat}(\alpha) \varepsilon_{m+k, k}(g)\left(\right.$ resp. $\left.\operatorname{Mat}(\alpha) \varepsilon_{m+k, k}(-g)\right)$, where $\beta_{i}^{\prime}=\beta_{i}$ if $i \neq k$, and $\beta_{k}^{\prime}$ is the embedding obtained after the deformation of $\beta_{k}$.

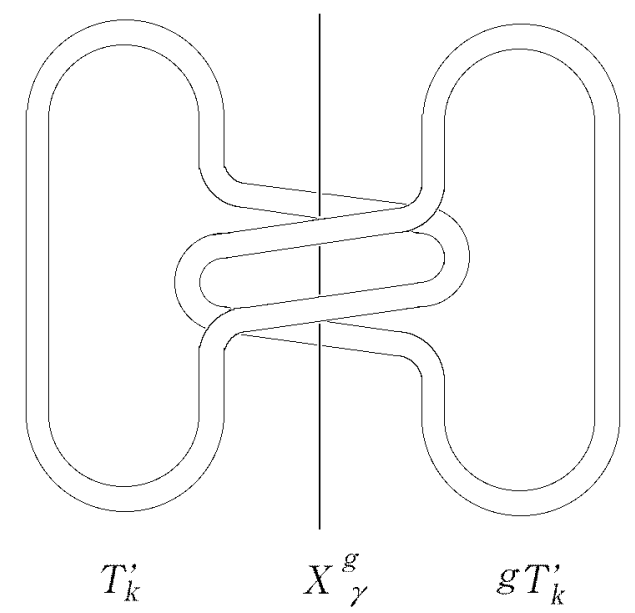

Figure 4.12

Proof. We may suppose $k=1$. We define an $R[G]$-homomorphism $\beta$ : $K_{1}(\partial G U ; R) \rightarrow K_{1}\left(\partial G U^{\prime} ; R\right)$ by

$$
\left.\beta\left(\begin{array}{c}
e_{1} \\
\vdots \\
e_{m} \\
f_{1} \\
f_{2} \\
\vdots \\
f_{m}
\end{array}\right)=\left(\begin{array}{c}
e_{1}^{\prime} \\
\vdots \\
e_{m}^{\prime} \\
\epsilon g e_{1}^{\prime}+f_{1}^{\prime} \\
f_{2}^{\prime} \\
\vdots \\
f_{m}^{\prime}
\end{array}\right)=\varepsilon_{m+1,1}(\epsilon g)\left(\begin{array}{c}
e_{1}^{\prime} \\
\vdots \\
e_{m}^{\prime} \\
f_{1}^{\prime} \\
\vdots \\
f_{m}^{\prime}
\end{array}\right)\right) .
$$

Then $\beta$ is an isomorphism $\left(K_{1}(\partial G U ; R), \lambda, \mu\right) \rightarrow\left(K_{1}\left(\partial G U^{\prime} ; R\right), \lambda^{\prime}, \mu^{\prime}\right)$. It holds that

$$
\beta\left(K_{2}\left(X_{0}, \partial G U ; R\right)\right)=K_{2}\left(X_{0}^{\prime}, \partial G U^{\prime} ; R\right)
$$


Define an endomorphism $\alpha^{\prime}$ of $K_{1}\left(\partial G U^{\prime} ; R\right)$ by

$$
\alpha^{\prime}\left(\begin{array}{c}
e_{1}^{\prime} \\
\vdots \\
e_{m}^{\prime} \\
f_{1}^{\prime} \\
\vdots \\
f_{m}^{\prime}
\end{array}\right)=\operatorname{Mat}(\alpha) \varepsilon_{m+1,1}(\epsilon g)\left(\begin{array}{c}
e_{1}^{\prime} \\
\vdots \\
e_{m}^{\prime} \\
f_{1}^{\prime} \\
\vdots \\
f_{m}^{\prime}
\end{array}\right) .
$$

Then $\alpha^{\prime}$ is an automorphism of $\left(K_{1}\left(\partial G U^{\prime} ; R\right), \lambda^{\prime}, \mu^{\prime}\right)$. Since $\alpha^{\prime}\left(e_{i}^{\prime}\right)=\beta \alpha\left(e_{i}\right)$ for all $i=1, \ldots, m$, we have

$$
\alpha^{\prime}\left(K_{2}\left(G U^{\prime}, \partial G U^{\prime} ; R\right)\right)=\beta \alpha\left(K_{2}(G U, \partial G U ; R)\right)=K_{2}\left(X_{0}^{\prime}, \partial G U^{\prime} ; R\right) .
$$

Hence, $\alpha^{\prime}$ is preferable and the surgery matrix $\operatorname{Mat}\left(\alpha^{\prime}\right)$ associated with $\alpha^{\prime}$ is $\operatorname{Mat}(\alpha) \varepsilon_{m+1,1}(\epsilon g)$.

Q.E.D.

Putting all together, we have proved Theorem 1.1.

One can prove Theorem 1.3 by a similar argument using the lemma below.

Let $a$ and $k$ be natural numbers satisfying $1 \leq k \leq m$. Let $\beta_{k}: S^{1} \times D^{2} \rightarrow$ $X$ be an embedding as before. Then, Twist ${ }_{a}\left(\beta_{k}\right): S^{1} \times D^{2} \rightarrow X$ is defined by

$$
\operatorname{Twist}_{a}\left(\beta_{k}\right)(x, y)=\beta_{k}\left(x, x^{a} y\right) \quad\left(x \in S^{1} \text { and } y \in D^{2}\right) .
$$

We call Twist ${ }_{a}\left(\beta_{k}\right)$ the a-times twisted embedding of $\beta_{k}$.

Lemma 4.13. Replacing $\beta_{k}$ by the $(-a)$-times twisted embedding $\operatorname{Twist}_{(-a)}\left(\beta_{k}\right)$ alters the surgery matrix $\operatorname{Mat}(\alpha)$ for $\left\{\beta_{1}, \ldots, \beta_{m}\right\}$ to a surgery matrix $\operatorname{Mat}\left(\alpha^{\prime}\right)$ for $\left\{\beta_{1}^{\prime}, \ldots, \beta_{m}^{\prime}\right\}$ such that $\operatorname{Mat}\left(\alpha^{\prime}\right)=\operatorname{Mat}(\alpha) \varepsilon_{m+k, k}(a)$, where $\beta_{i}^{\prime}=\beta_{i}$ if $i \neq k$ and $\beta_{k}^{\prime}=\operatorname{Twist}_{(-a)}\left(\beta_{k}\right)$.

Proof. Without loss of generality, we may assume that $k=1$. Since $\operatorname{Im} \beta_{1}^{\prime}=\operatorname{Im} \beta_{1}$, we may use $U^{\prime}=U$ and $X_{0}^{\prime}=X_{0}$ to obtain a surgery matrix. Define an $R[G]$-endomorphism $\alpha^{\prime}$ of $K_{1}\left(\partial G U^{\prime} ; R\right)$ by

$$
\alpha^{\prime}\left(\begin{array}{c}
e_{1}^{\prime} \\
\vdots \\
e_{m}^{\prime} \\
f_{1}^{\prime} \\
\vdots \\
f_{m}^{\prime}
\end{array}\right)=\alpha\left(\begin{array}{c}
e_{1} \\
\vdots \\
e_{m} \\
f_{1} \\
\vdots \\
f_{m}^{\prime}
\end{array}\right)
$$


Then we obtain

$$
\alpha^{\prime}\left(\begin{array}{c}
e_{1}^{\prime} \\
\vdots \\
e_{m}^{\prime} \\
f_{1}^{\prime} \\
\vdots \\
f_{m}^{\prime}
\end{array}\right)=\operatorname{Mat}(\alpha)\left(\begin{array}{c}
e_{1}^{\prime} \\
\vdots \\
e_{m}^{\prime} \\
a e_{1}^{\prime}+f_{1}^{\prime} \\
\vdots \\
f_{m}^{\prime}
\end{array}\right)=\operatorname{Mat}(\alpha) \varepsilon_{m+1,1}(a)\left(\begin{array}{c}
e_{1}^{\prime} \\
\vdots \\
e_{m}^{\prime} \\
f_{1}^{\prime} \\
\vdots \\
f_{m}^{\prime}
\end{array}\right),
$$

because $e_{1}^{\prime}=e_{1}$ and $f_{1}^{\prime}=f_{1}+(-a) e_{1}$ in $K_{1}\left(\partial U^{\prime} ; R\right)=K_{1}(\partial U ; R)$. The endomorphism $\alpha^{\prime}$ is an automorphism of $\left(K_{1}\left(\partial G U^{\prime} ; R\right), \lambda^{\prime}, \mu^{\prime}\right)$. Furthermore

$$
\alpha^{\prime}\left(K_{2}\left(G U^{\prime}, \partial G U^{\prime} ; R\right)\right)=K_{2}\left(X_{0}^{\prime}, \partial G U^{\prime} ; R\right) .
$$

Thus this $\alpha^{\prime}$ is preferable for $\left\{\beta_{1}^{\prime}, \ldots, \beta_{m}^{\prime}\right\}$. The surgery matrix Mat $\left(\alpha^{\prime}\right)$ satisfies $\operatorname{Mat}\left(\alpha^{\prime}\right)=\operatorname{Mat}(\alpha) \varepsilon_{m+1,1}(a)$.

Q.E.D.

\section{$\S 5$. Singular Sets of Actions of $A_{5}$ on Homology 3-spheres}

Let $A_{5}$ be the alternating group on five letters, and $\mathrm{SO}(3)$ the special orthogonal group of degree three. For a nontrivial homomorphism $\rho: A_{5} \rightarrow$ $\mathrm{SO}(3)$, the Poincaré sphere $\Sigma=\Sigma(\rho)$ is defined to be the space of left cosets, $\mathrm{SO}(3) / \rho\left(A_{5}\right)$. A smooth action of $A_{5}$ on the Poincaré sphere $\Sigma$ is naturally given. That is,

$$
A_{5} \times \Sigma \rightarrow \Sigma ;\left(g, h \rho\left(A_{5}\right)\right) \mapsto \rho(g) h \rho\left(A_{5}\right) .
$$

We call this action the standard action of $A_{5}$ on $\Sigma$. This standard action on the Poincaré sphere is investigated in [9]. In particular, there are two $A_{5}$ diffeomorphism (or $A_{5}$-homotopy) types of the Poincaré spheres. They are decided by the characters of homomorphisms $A_{5} \rightarrow \mathrm{SO}(3)$. The purpose of this section is to study the $G$-homeomorphism types of the singular sets of smooth actions of $A_{5}$ on homology spheres of dimension 3 with exactly one fixed point, and to prove Theorem 5.5 below.

We denote the cyclic group of order $m$ by $C_{m}$ and the dihedral group of order $2 m$ by $D_{2 m}$. We also denote by $A_{4}$ the alternating group on four letters, which is isomorphic to the tetrahedral group. For elements $g_{1}, g_{2}, \ldots, g_{n}$ of $A_{5}$, the subgroup of $A_{5}$ generated by $g_{1}, g_{2}, \ldots, g_{n}$ is denoted by $\left\langle g_{1}, g_{2}, \ldots, g_{n}\right\rangle$.

Definition 5.1. We set $x=(1,2)(3,4), y=(3,5,4), z=(1,2,3,5,4)$ and $u=(1,3)(2,4)$ in $A_{5}$. The subgroup $\langle x\rangle$ is properly contained in the 
following seven subgroups.

$$
\begin{aligned}
& \langle x, u\rangle\left(\cong D_{4}\right), \quad\langle x, y\rangle\left(\cong D_{6}\right), \quad\langle x, u y u\rangle\left(\cong D_{6}\right) \\
& \langle x, z\rangle\left(\cong D_{10}\right), \quad\langle x, u z u\rangle\left(\cong D_{10}\right), \quad\left\langle x, z^{2} u z\right\rangle\left(\cong A_{4}\right) \text { and } A_{5}
\end{aligned}
$$

The subgroup $\left\langle x, z^{2} u z\right\rangle$ contains $\langle x, u\rangle$. Throughout this section, unless otherwise stated, the above elements $x, y, z$ and $u$ are fixed as above and we use the notation

$$
\begin{gathered}
C_{2}=\langle x\rangle, C_{3}=\langle y\rangle, C_{5}=\langle z\rangle \\
D_{4}=\langle x, u\rangle, D_{6}=\langle x, y\rangle, D_{10}=\langle x, z\rangle \text { and } A_{4}=\left\langle x, z^{2} u z\right\rangle .
\end{gathered}
$$

Any subgroup of $A_{5}$ is conjugate to one of the groups in the next figure (cf. $[4$, p.10]).

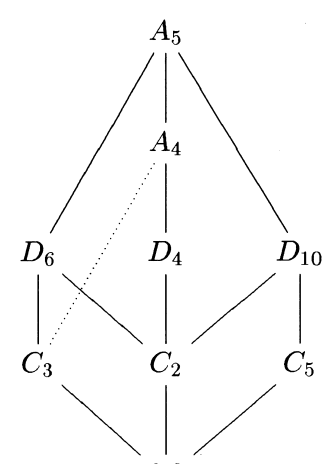

$\{e\}$

Figure 5.2

Definition 5.3. We denote by $\mathfrak{M}^{3}$ the family of closed, oriented, 3dimensional, smooth $A_{5}$-manifolds $X$ satisfying the following conditions (5.3.1)(5.3.4).

(5.3.1) $\left|X^{A_{5}}\right|=1$.

(5.3.2) $X^{H}=X^{K}$ whenever $H \subset K \subset A_{5}, H \cong D_{4}$ and $K \cong A_{4}$.

(5.3.3) $\left|X^{H}\right|=2$ whenever $H \subset A_{5}$ and $H \cong D_{2 m}$ for some $m=2,3$ or 5 .

(5.3.4) $X^{H}$ is diffeomorphic to $S^{1}$ whenever $H \subset A_{5}$ and $H \cong C_{m}$ for some $m=2,3$ or 5 . 
It is well-known that if $X$ is a 3-dimensional homology sphere having a smooth action of $A_{5}$ with exactly one fixed point, then $X$ lies in $\mathfrak{M}^{3}$. The Poincaré sphere $\Sigma$ is a homology sphere and the standard action of $A_{5}$ on it has exactly one fixed point. Thus, $\Sigma$ belongs to $\mathfrak{M}^{3}$.

Let $X \in \mathfrak{M}^{3}$. For each subgroup $H$ of $A_{5}$ such that $H \cong A_{5}, A_{4}, D_{10}$ or $D_{6}$, there exists a unique point $p(H) \in X$ with isotropy subgroup $H$. Imagine that we walk on the circle $X^{C_{2}}$ starting from and ending at $p\left(A_{5}\right)$. Since $u x=x u$, the action of $u$ gives a diffeomorphism of $X^{C_{2}}$ fixing $p\left(A_{5}\right)$ and $p\left(A_{4}\right)$ and interchanging $p\left(D_{2 m}\right)$ and $p\left(u D_{2 m} u\right)$ for $m=3$ and 5 . Hence, on $X^{C_{2}}$, we must meet the intersection points $p(H)$ in one of the following order. (Note: In each case, we do not specify a direction.)

(1)

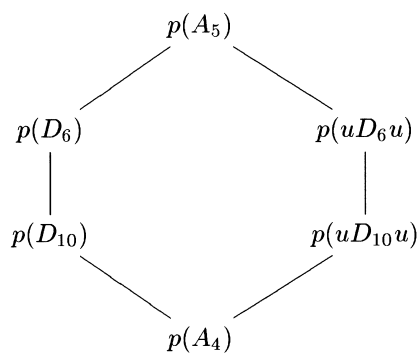

(3)

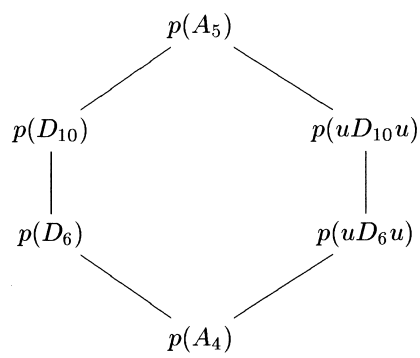

(2)

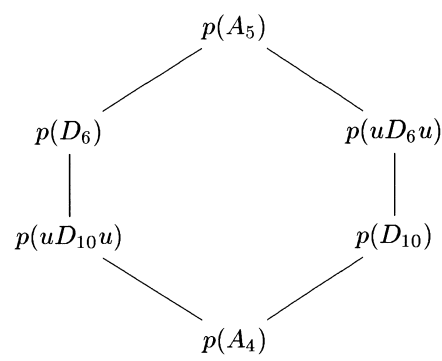

(4)

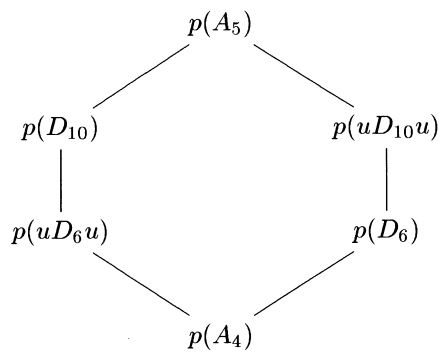

Definition 5.4. Let $C_{2}, A_{4}, D_{6}, D_{10}, u D_{6} u$ be the specified subgroups of $A_{5}$ as in Definition 5.1. We say that $X \in \mathfrak{M}^{3}$ is of type $\left(A_{5}-D_{6}-D_{10}-A_{4}\right)$, $\left(A_{5}-D_{6}-u D_{10} u-A_{4}\right),\left(A_{5}-D_{10}-D_{6}-A_{4}\right)$ or $\left(A_{5}-D_{10}-u D_{6} u-A_{4}\right)$ according as the figure of $X^{C_{2}}$ is (1), (2), (3) or (4) above.

The type of the Poincaré sphere with standard action is determined in [9, Theorem 1.13]. Let $\rho: A_{5} \rightarrow \mathrm{SO}(3)$ be a nontrivial representation. Then the Poincaré sphere $\Sigma(\rho)$ with standard action is of type $\left(A_{5}-D_{6}-u D_{10} u-A_{4}\right)$ 
if $\chi_{\rho}(z)=(1+\sqrt{5}) / 2$, and $\left(A_{5}-D_{6}-D_{10}-A_{4}\right)$ if $(1-\sqrt{5}) / 2$, where $\chi_{\rho}$ is the character associated with $\rho$.

Theorem 5.5. For any nontrivial real representation $\rho: A_{5} \rightarrow \mathrm{SO}(3)$ of $A_{5}$ and any type $\gamma$ of the singular set, there exists a smooth action $X$ of $A_{5}$ on a 3-dimensional homology sphere with exactly one fixed point such that the tangential representation at the unique fixed point is isomorphic to $V(\rho)$, the type of the singular set of $X$ is $\gamma$, and $X$ is $A_{5}$-cobordant to $\Sigma(\rho)$, where $V(\rho)$ is the $A_{5}$-module associated with $\rho$.

Proof. The tangential representation of $\Sigma(\rho)$ at the unique $A_{5}$-fixed point $p\left(A_{5}\right)$ is isomorphic to $V(\rho)$. Take a closed $A_{5}$-disk neighborhood $D(V(\rho))$ around $p\left(A_{5}\right)$ in $\Sigma(\rho)$. Pinching the outside of $\operatorname{Int} D(V(\rho))$, we obtain an $A_{5-}$ map $f^{\prime \prime}: \Sigma(\rho) \rightarrow Y$ of degree one, where $Y=S(\mathbb{R} \oplus V(\rho))$ and $f^{\prime \prime}\left(p\left(A_{5}\right)\right)=$ $(1,0)$. It is easy to see that $f^{\prime \prime}$ can be converted by $A_{5}$-surgeries of isotropy type $\left(C_{2}\right)$ to an $A_{5}$-map $f: X \rightarrow Y$ such that $X \in \mathfrak{M}^{3}$ is of type $\gamma$ (by the same argument as [9, Proof of Lemma 2.4]). Since the employed surgeries are of isotropy type $\left(C_{2}\right)$, they do not change the fixed point sets of $A_{5}, A_{4},\left(D_{4},\right)$ $D_{6}$ and $D_{10}$. By Theorem 1.3, the $A_{5}$-surgery obstruction $\sigma(f)$ to converting $f: X \rightarrow Y$ to a homology equivalence keeping $f_{s}: X_{s} \rightarrow Y_{s}$ fixed, lies in the group $W_{3}\left(\mathbb{Z}\left[A_{5}\right]\right.$, max; triv). Since by [2, Corollary 6$]$ this group is trivial, we can perform $A_{5}$-surgery, and obtain a homology equivalence $f^{\prime}: X^{\prime} \rightarrow Y$. Here $X^{\prime} \in \mathfrak{M}^{3}$ is the $A_{5}$-manifold required in Theorem 5.5.

Q.E.D.

\section{References}

[1] Bak, A., K-Theory of Forms, Princeton University Press, Princeton, 1981.

[2] Bak, A. and Morimoto, M., Equivariant surgery and applications, Proceedings of Topology Hawaii 1990 (ed K. Dovermann) p.13-25, World Scientific Publ., Singapore, 1992.

[3] Cohen, M. M., A Course in Simple Homotopy Theory, Graduate Texts in Math., 21 Springer, Berlin-Heidelberg-New York, 1973.

[4] tom Dieck, T., Transformation Groups and Representation Theory, Lect. Notes in Math., 766 Springer Berlin-Heidelberg-New York, 1979.

[5] Morimoto, M., Bak groups and equivariant surgery, K-Theory, 2 (1989), 465-483.

[6] - Bak groups and equivariant surgery II, K-Theory, 3 (1990), 505-521.

[7] — A geometric quadratic form of 3-dimensional normal maps, Top. Its Appl., 83 (1998), 77-102.

[8] Morimoto, M. and Iizuka, K., Extendibility of $G$-maps to pseudo-equivalences to finite G-CW-complexes whose fundamental groups are finite, Osaka J. Math., 21 (1984), 5969.

[9] Morimoto, M. and Uno, K., Remarks on one fixed point $A_{5}$-actions on homology spheres, Proceedings of Algebraic Topology Poznań, 1989 (eds S. Jackowski, B. Oliver and K. Pawałowski), Lect. Notes in Math., 1474 (1991), 337-364, Springer, Berlin-HeidelbergNew York. 